ESAIM: PROCEEDINGS, April 2007, Vol.17, 26-49

Alain Piétrus \& Michel H. Geoffroy, Editors

\title{
EXACT CONTROLLABILITY OF AN AEROACOUSTIC MODEL
}

\author{
L. Cot ${ }^{1}$, J.-P. Raymond ${ }^{2}$ and J. VAnCostenoble ${ }^{3}$
}

\begin{abstract}
We study the exact controllability of a fluid-structure model. The fluctuations of velocity and pressure in the fluid are described by a potential, and the structure is a membrane located in a part $\Gamma_{s}$ of the boundary of the domain $\Omega$. The potential $\phi$ and the transverse displacement $z$ satisfy a coupled system of two wave equations, one in the domain $\Omega \times(0, T)$, the other one in the boundary $\Gamma_{s} \times(0, T)$. Taking two boundary controls, the first one in a boundary condition satisfied by the potential, and the second one in a boundary condition of the structure equation, we identify the space of controllable initial conditions when the geometrical controllability conditions are satisfied. As in the case of the so-called Helmholtz fluid-structure model [10], the difficulty in the treatement of the observability inequalities, in the definition of very weak solutions, and in the proof of controllability result, comes from the coupling terms of the system. To overcome these difficulties, we show that the variant introduced in [10] of the classical Hilbert Uniqueness Method can be adapted to the aeroacoustic model we consider.
\end{abstract}

\section{INTRODUCTION}

In this paper, we are interested in the control of aeroacoustic models studied in [9], [6], [7], [1], describing acoustic wave propagation in a cavity $\Omega$ filled by a fluid flow. In these models, the pressure (the fluctuation from the mean pressure at equilibrium) and the fluctuations of the velocity field are related to a velocity potential $\phi$ by $p=\rho_{f} \phi^{\prime}$ and $v=-\nabla \phi$, where $\rho_{f}$ is the density of the fluid at equilibrium. The potential $\phi$ satisfies the undamped wave equation:

$$
\phi^{\prime \prime}-c_{f}^{2} \Delta \phi=0 \quad \text { in } \Omega \times(0, T)
$$

where $c_{f}^{2}$ is the speed of sound in the fluid. A part $\Gamma_{s}$ of the boundary of $\Omega$ is occupied by a structure. In [9], [6], [7], [1], and also in the present paper, the structure is a membrane. The structure could also be a beam for a 2D model as in [3], a plate or a shell in a 3D domain [2]. In the following, let us denote by $z$ the transverse displacement of the structure. The common feature of these models is that the continuity condition of velocity on $\Gamma_{s}$ is:

$$
\partial_{n} \phi=z^{\prime} \quad \text { on } \quad \Gamma_{s} \times(0, T),
$$

where $\partial_{n} \phi$ is the normal derivative of $\phi$ at the boundary $\Gamma$ of $\Omega$, and the equation of the transverse displacement of the structure involves

$$
-\frac{\rho_{f}}{\rho_{s}} \phi^{\prime}
$$

as a source term in the equation. In the case of a membrane the transverse displacement satisfies a wave equation $z^{\prime \prime}-c_{s}^{2} \Delta_{s} z=-\frac{\rho_{f}}{\rho_{s}} \phi^{\prime} \quad$ in $\Gamma_{s} \times(0, T)$, where $c_{s}^{2}$ is the speed of sound in the structure, $\rho_{s}$ is the density of the

\footnotetext{
1 Laboratoire MIP, UMR CNRS 5640, Université Paul Sabatier, 31062 Toulouse Cedex 4, France, cot@mip.ups-tlse.fr

${ }^{2}$ Laboratoire MIP, UMR CNRS 5640, Université Paul Sabatier, 31062 Toulouse Cedex 4, France, raymond@mip.ups-tlse.fr

3 Laboratoire MIP, UMR CNRS 5640, Université Paul Sabatier, 31062 Toulouse Cedex 4, France, vancoste@mip.ups-tlse.fr
} 
structure, and $\Delta_{s}$ is the Laplace operator in $\Gamma_{s}$ ( $\Gamma_{s}$ is supposed to be flat). The model is completed by other boundary conditions on $\Gamma \backslash \Gamma_{s}$ for the potential $\phi$, for example $\partial_{n} \phi=0$ on a part of $\left(\Gamma \backslash \Gamma_{s}\right) \times(0, T)$ and $\phi=$ 0 on an other part of $\left(\Gamma \backslash \Gamma_{s}\right) \times(0, T)$, and a boundary condition on $\partial \Gamma_{s}$ for the transverse displacement $z$, for example $z=0$ on $\partial \Gamma_{s}$. We would like to control the complete model - including the boundary conditions - by means of a control in the structure equation, or in the boundary conditions of the potential, or by both controls one on the structure and the other one on the fluid. The case of a control on $\Gamma_{s}$ in the structure equation has been considered in [9], [7] and [1]. The results in [9] show that, when $\Omega$ is a rectangle, only initial conditions satisfying a very specific decay of Fourier modes may be controlled. An approximate controllability result is stated in [7] for particular initial conditions generating waves localized near the structure and called Stoneley waves. In both references, [9] and [7], the controllability is studied by Fourier analysis. In [1], for very particular domains, a controllability result is proved for initial conditions corresponding to the space of finite energy solutions with a control in $\left(H^{1}\left(0, T ; L^{2}\left(\Gamma_{s}\right)\right)\right)^{\prime}$, acting only as a distributed term in the structure equation. For other domains, an other controllability result is proved with both controls, one acting as a distributed term in the structure equation and belonging to $L^{2}\left(0, T ; H^{-\alpha}\left(\Gamma_{s}\right)\right)$ for some $1 / 4 \leq \alpha \leq 1 / 3$, and the other one acting in a Neumann boundary condition for the fluid and belonging to $L^{2}\left(0, T ; L^{2}\left(\Gamma \backslash \Gamma_{s}\right)\right.$ ) (see [1, Theorem $\left.\left.1 \mathrm{~b}\right]\right)$. To the best of our knowledge the case of a control acting only on a part of the boundary $\Gamma \backslash \Gamma_{s}$ for the fluid, and another one acting in the structure equation (either as a distributed control or a boundary control), seems to be open.

In this paper, we would like to answer the following question:

Can one adapt to the fluid-structure models above described the techniques developed in [8] for the wave equation?

In other words, let us take a control in the structure equation (or in the boundary conditions of the structure equation), and a control in a part of the boundary conditions of the fluid equation (either in a Neumann boundary condition, or in a Dirichlet boundary condition), can we affirm that we are able to control the coupled system ?

At first sight, the answer seems to be obvious: if we control both equations, it should be easy to control the coupled system. We are going to see that the situation is complicated by the presence of the two coupling terms: $z^{\prime}$ in the boundary conditions of $\phi$, and $-\frac{\rho_{f}}{\rho_{s}} \phi^{\prime}$ in the structure equation. Indeed, $\operatorname{denoting}$ by $Z=$ $V \times L^{2}(\Omega) \times H_{0}^{1}\left(\Gamma_{s}\right) \times L^{2}\left(\Gamma_{s}\right)$ the space of initial conditions of finite energy solutions of the coupled system, we explain in section 2 why we cannot define weak solutions of the coupled system for initial data in $L^{2}(\Omega) \times$ $V^{\prime} \times L^{2}\left(\Gamma_{s}\right) \times H^{-1}\left(\Gamma_{s}\right)$. This particular feature has already been met with an other simplified fluid-structure model [10]. To overcome this difficulty, following [10], we have to introduce an other space of initial data, denoted by $Y$ throughout the paper, in order to adapt the Hilbert Uniqueness Method to the fluid-structure model that we consider. Let us now precisely describe the fluid-structure model we want to control. We suppose that $\Omega$ is a simply connected bounded domain in $\mathbb{R}^{2}$, its boundary $\Gamma$ is split into two parts: a flat part $\Gamma_{s}$ corresponding to the membrane, and the complementary part $\Gamma_{0}$ on which we impose a Dirichlet boundary condition. The uncontrolled model is the following one:

$$
\begin{cases}\phi^{\prime \prime}-c_{f}^{2} \Delta \phi=0 & \text { in } \Omega \times(0, T), \\ \phi=0 & \text { on } \Gamma_{0} \times(0, T), \\ \partial_{n} \phi=z^{\prime} & \text { on } \Gamma_{s} \times(0, T), \\ \phi(0)=\phi^{0} \quad \text { and } \quad \phi^{\prime}(0)=\phi^{1} & \text { in } \Omega, \\ z^{\prime \prime}-c_{s}^{2} \Delta_{s} z=-\frac{\rho_{\mathbf{f}}}{\rho_{\mathbf{s}}} \phi^{\prime} & \text { in } \Gamma_{s} \times(0, T), \\ z(0, t)=z(L, t)=0 & \text { in }(0, T), \\ z(0)=z^{0} \text { and } \quad z^{\prime}(0)=z^{1} & \text { in } \Gamma_{s},\end{cases}
$$

where $\Omega=] 0, L[\times] 0, \ell\left[, \Gamma_{s}=\right] 0, L\left[\times\{0\}\right.$ and $\Gamma_{0}=\Gamma \backslash \Gamma_{s}$.

In this paper, we consider the case where $\Omega$ is a rectangular domain, in orde $r$ to underline the difficulties due to 
the fluid-structure interactions. The only difficulty to extend our controllability result to other kinds of domains is in the proof of the multiplier lemma (see Lemma 5.1). In the case of a rectangular domain, the solution is regular enough to justify the computations in the proof of Lemma 5.1. For others domains, these computations have to be justified.

The main objective of this work is to extend the well-known boundary controllability result of the wave equation stated in [8, Chapter 1, Theorem 6.1]. Here, we take two boundary controls: $u_{1}$ acting in a boundary condition of the fluid equation (i.e. on a part of $\Gamma_{0}$ ), and $u_{2}$ acting in a boundary condition of the structure equation (i.e. on a part of $\left.\partial \Gamma_{s}=\{(0,0) ;(L, 0)\}\right)$. In order to obtain sharp results, we do not consider $u_{1}$ and $u_{2}$ acting everywhere on $\Gamma_{0}$ and on $\partial \Gamma_{s}$. According to the well-known results concerning the wave equation (the so-called optic ray condition, see [4]), it is natural to consider $u_{1}$ acting on $\Gamma_{c}$, where $\Gamma_{c}$ is composed of the upper side and the right-hand side of $\partial \Omega$, and $u_{2}$ acting on the point $x=(L, 0)$ (see figure 2 ). More precisely, we consider

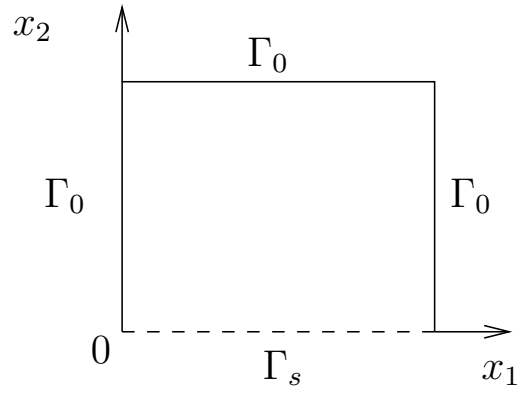

Figure 1

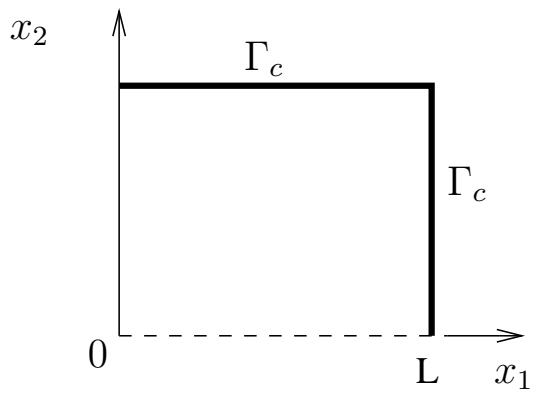

Figure 2

the controlled system:

$$
\begin{cases}\phi^{\prime \prime}-c_{f}^{2} \Delta \phi=0 & \text { in } \Omega \times(0, T), \\ \phi=u_{1} & \text { on } \Gamma_{c} \times(0, T), \\ \phi=0 & \text { on }\left(\Gamma_{0} \backslash \Gamma_{c}\right) \times(0, T), \\ \partial_{n} \phi=z^{\prime} & \text { on } \Gamma_{s} \times(0, T), \\ \phi(0)=\phi^{0} \quad \text { and } \quad \phi^{\prime}(0)=\phi^{1} & \text { in } \Omega, \\ z^{\prime \prime}-c_{s}^{2} \Delta_{s} z=-\frac{\rho_{f}}{\rho_{s}} \phi^{\prime} & \\ z(0, t)=0 \quad \text { and } \quad z(L, t)=u_{2}(t) & \text { in } \Gamma_{s} \times(0, T), \\ z(0)=z^{0} \text { and } \quad z^{\prime}(0)=z^{1} & \text { in }(0, T),\end{cases}
$$

As already mentioned above the boundary condition $\phi=u_{1}$, for the fluid equation, may seem to be unrealistic from the physical view point. A more realistic one - a control in a Neumann boundary condition - will be considered in a forthcoming paper, but the analysis will be there more delicate (see [5]). Our main objective is to explain how the Hilbert Uniqueness Method may be adapted to some fluid-structure models. We are going to see that some tools introduced in [10] for a simplified model may be extended to more sophisticated ones. If we summarize, we are interested in the control problem:

Find $T>0$ and a space of initial conditions $E_{i c}$ such that, for all $\left(\phi^{0}, \phi^{1}, z^{0}, z^{1}\right) \in E_{i c}$, there exists a pair of controls $\left(u_{1}, u_{2}\right) \in L^{2}\left(\Gamma_{c} \times(0, T)\right) \times L^{2}(0, T)$, for which the solution (in a sense to be defined) $(\phi, z)$ of system (2) obeys:

$$
\phi(T)=\phi^{\prime}(T)=0 \text { in } \Omega \text { and } \quad z(T)=z^{\prime}(T)=0 \text { in } \Gamma_{s} .
$$


Accordingly, the paper is organized as follows. Our main results are stated in section 2. We study the wellposedness of the different systems appearing in the Hilbert Uniqueness Method in sections 3 and 4 . In section 5, we present the different steps of the Hilbert Uniqueness Method and the proof of the controllability result. In appendix, we establish the three main ingredients, that is, the multiplier lemma, the direct inequality and the inverse inequality.

\section{Main Results}

Throughout the paper, we use the following notation:

$Q=\Omega \times(0, T), \Sigma=\Gamma \times(0, T), \Sigma_{0}=\Gamma_{0} \times(0, T), \Sigma_{s}=\Gamma_{s} \times(0, T), \Sigma_{c}=\Gamma_{c} \times(0, T), \sigma_{s}=\partial \Gamma_{s} \times(0, T)$.

\subsection{Well-posedness of the homogeneous problem}

We introduce the space

Let us set $\alpha=c_{f}^{2} \frac{\rho_{s}}{\rho_{f}}$. The space

$$
V=\left\{\phi \in H^{1}(\Omega)|\phi|_{\Gamma_{0}}=0\right\}
$$

equipped with the inner product

$$
Z=V \times L^{2}(\Omega) \times H_{0}^{1}\left(\Gamma_{s}\right) \times L^{2}\left(\Gamma_{s}\right),
$$

$$
\left(\left(\phi_{0}, \phi_{1}, z_{0}, z_{1}\right),\left(\hat{\phi}_{0}, \hat{\phi}_{1}, \hat{z}_{0}, \hat{z}_{1}\right)\right)_{Z}=c_{f}^{2} \int_{\Omega} \nabla \phi_{0} \nabla \hat{\phi}_{0}+\int_{\Omega} \phi_{1} \hat{\phi}_{1}+\alpha c_{s}^{2} \int_{\Gamma_{s}} \nabla_{s} z_{0} \nabla_{s} \hat{z}_{0}+\alpha \int_{\Gamma_{s}} z_{1} \hat{z}_{1},
$$

and the associated norm

$$
\left\|\left(\phi_{0}, \phi_{1}, z_{0}, z_{1}\right)\right\|_{Z}:=\left(c_{f}^{2}\left\|\phi_{0}\right\|_{V}^{2}+\left\|\phi_{1}\right\|_{L^{2}(\Omega)}^{2}+\alpha c_{s}^{2}\left\|z_{0}\right\|_{H_{0}^{1}\left(\Gamma_{s}\right)}^{2}+\alpha\left\|z_{1}\right\|_{L^{2}\left(\Gamma_{s}\right)}^{2}\right)^{1 / 2}
$$

is a Hilbert space. Observe that $\nabla_{s} z$ is nothing but $\frac{\mathrm{d} z}{\mathrm{~d} x_{1}}$. Let us define the energy of regular solutions $(\phi, z)$ of system (1) by:

$$
E(t):=\frac{1}{2} \int_{\Omega}\left(\phi^{\prime 2}+c_{f}^{2}|\nabla \phi|^{2}\right)+\frac{\alpha}{2} \int_{\Gamma_{s}}\left(z^{\prime 2}+c_{s}^{2}\left|\nabla_{s} z\right|^{2}\right) .
$$

Classical computations show that regular solutions $(\phi, z)$ of $(1)$ satisfy:

$$
E(t)=E(0) \quad \text { for all } t \in[0, T]
$$

To study system (1) we rewrite it as a first order evolution equation. Setting $U=\left(\phi, \phi^{\prime}, z, z^{\prime}\right)$, the system (1) may be rewritten in the form:

where

$$
\frac{d U}{d t}=A U \quad \text { in } \quad(0, T), \quad U(0)=U_{0}
$$

$$
A U:=A\left(\begin{array}{c}
\phi_{0} \\
\phi_{1} \\
z_{0} \\
z_{1}
\end{array}\right)=\left(\begin{array}{c}
\phi_{1} \\
c_{f}^{2} \Delta \phi_{0} \\
z_{1} \\
c_{s}^{2} \Delta_{s} z_{0}-\frac{\rho_{f}}{\rho_{s}} \phi_{1}
\end{array}\right), \quad U_{0}:=\left(\begin{array}{c}
\phi^{0} \\
\phi^{1} \\
z^{0} \\
z^{1}
\end{array}\right)
$$

and the domain of $A$ in $Z$ is defined by:

$$
D(A)=\left\{\left(\phi_{0}, \phi_{1}, z_{0}, z_{1}\right) \in Z \mid \Delta \phi_{0} \in L^{2}(\Omega), \phi_{1} \in V, z_{0} \in H^{2}\left(\Gamma_{s}\right), z_{1} \in H_{0}^{1}\left(\Gamma_{s}\right), \text { and } \partial_{n} \phi_{0}=z_{1} \text { on } \Gamma_{s}\right\}
$$

Let us give a smoothness result, which is crucial in the following to justify all of our computations. 
Theorem 1.1. Let $\left(\phi_{0}, \phi_{1}, z_{0}, z_{1}\right)$ be given in $D(A)$. Then $\phi_{0} \in H^{2}(\Omega)$.

Proof. Let $\left(\phi_{0}, \phi_{1}, z_{0}, z_{1}\right)$ be given in $D(A)$. Then $\phi_{0}$ satisfies:

$$
-\Delta \phi_{0} \in L^{2}(\Omega), \quad \phi_{0}=0 \quad \text { on } \quad \Gamma_{0}, \quad \partial_{n} \phi_{0}=z_{1} \quad \text { on } \quad \Gamma_{s} .
$$

Since $z_{1}$ belongs to $H_{0}^{1}\left(\Gamma_{s}\right)$ and $\Omega$ is a rectangular domain, it can be shown that $\phi_{0} \in H^{2}(\Omega)$.

Next, proving that $(A, D(A))$ is the infinitesimal generator of a strongly continuous unitary group (see Lemma 2.1 ), we can state the following theorem.

Theorem 1.2. (i) For all $\left(\phi^{0}, \phi^{1}, z^{0}, z^{1}\right) \in Z$, the system (1) admits a unique weak solution $\left(\phi, \phi^{\prime}\right.$, $\left.z, z^{\prime}\right) \in \mathcal{C}([0, T] ; Z)$ which satisfies the estimate:

$$
\left\|\left(\phi, \phi^{\prime}, z, z^{\prime}\right)\right\|_{\mathcal{C}([0, T] ; Z)} \leq C\left\|\left(\phi^{0}, \phi^{1}, z^{0}, z^{1}\right)\right\|_{Z} .
$$

(ii) If moreover $\left(\phi^{0}, \phi^{1}, z^{0}, z^{1}\right) \in D(A)$, the solution obeys:

$$
\left\|\left(\phi, \phi^{\prime}, z, z^{\prime}\right)\right\|_{\mathcal{C}([0, T] ; D(A))} \leq C\left\|\left(\phi^{0}, \phi^{1}, z^{0}, z^{1}\right)\right\|_{D(A)} .
$$

Remark 1.3. Let us remark that Theorems 1.2 and 1.1 imply that the solution $(\phi, z)$ of $(1)$ associated with initial data $\left(\phi^{0}, \phi^{1}, z^{0}, z^{1}\right) \in D(A)$ satisfies: $(\phi, z) \in C\left([0, T] ; H^{2}(\Omega) \times H^{2}\left(\Gamma_{s}\right)\right)$.

We would like to extend to system (1) the well known controllability result of the wave equation with a Dirichlet control [8]. For that it should be natural to study the system (1) with initial conditions in $L^{2}(\Omega) \times$ $V^{\prime} \times L^{2}\left(\Gamma_{s}\right) \times H^{-1}\left(\Gamma_{s}\right)$. Unfortunately, because of the coupling term $-\frac{\rho_{f}}{\rho_{s}} \phi^{\prime}$ in the equation in $\Gamma_{s} \times(0, T)$, we cannot define solutions to system (1) for initial conditions in this space.

To extend the notion of solutions to system (1), we can use the extrapolation method. We denote by $\left(A^{*}, D\left(A^{*}\right)\right)$ the adjoint of $(A, D(A))$ with respect to the pivot space $Z$, and by $\left(D\left(A^{*}\right)\right)^{*}$ the dual of $D\left(A^{*}\right)$ with respect to the pivot space $Z$. We verify that $D(A)=D\left(A^{*}\right)$, and we have

$$
D(A) \hookrightarrow Z \hookrightarrow\left(D\left(A^{*}\right)\right)^{*}
$$

with dense and continuous imbeddings. The strongly continuous group $\left(e^{t A}\right)_{t \in \mathbb{R}}$ on $Z$ may be extended to a strongly continuous group on $\left(D\left(A^{*}\right)\right)^{*}$. We still denote it by $\left(e^{t A}\right)_{t \in \mathbb{R}}$. Following the approach introduced in [10] for the Helmholtz model, we can show that

$$
\left(\phi^{0}, \phi^{1}, z^{0}, z^{1}\right) \mapsto\left\|\left(\phi^{0}, \phi^{1}, z^{0}, z^{1}\right)\right\|_{Y}=\left(\left\|\phi^{0}\right\|_{L^{2}(\Omega)}^{2}+\left\|\phi^{1}\right\|_{V^{\prime}}^{2}+\left\|z^{0}\right\|_{L^{2}\left(\Gamma_{s}\right)}^{2}+\left\|\alpha z^{1}+c_{f}^{2} \phi^{0}\right\|_{H^{-1}\left(\Gamma_{s}\right)}^{2}\right)^{1 / 2}
$$

is a norm on $Z$ equivalent to the norm

$$
\left\|\left(\phi^{0}, \phi^{1}, z^{0}, z^{1}\right)\right\|_{\left(D\left(A^{*}\right)\right)^{*}}=\sup _{\left(\psi^{0}, \psi^{1}, w^{0}, w^{1}\right) \in D\left(A^{*}\right)} \frac{\left(\left(\phi^{0}, \phi^{1}, z^{0}, z^{1}\right),\left(\psi^{0}, \psi^{1}, w^{0}, w^{1}\right)\right)_{Z}}{\left\|\left(\psi^{0}, \psi^{1}, w^{0}, w^{1}\right)\right\|_{D\left(A^{*}\right)}} .
$$

(Such a result can be proved as in [5]). Thus the closure of $Z$ in this norm $\|\cdot\|_{Y}$, denoted by $Y$, can be identified algebraically and topologically with $\left(D\left(A^{*}\right)\right)^{*}$. Therefore we can state the following result.

Theorem 1.4. For all $\left(\phi^{0}, \phi^{1}, z^{0}, z^{1}\right) \in Y$, system (1) admits a unique weak solution $(\phi, z)$ (in the sense of semigroup theory) which belongs to $\mathcal{C}([0, T] ; Y)$ and satisfies

$$
\left\|\left(\phi, \phi^{\prime}, z, z^{\prime}\right)\right\|_{\mathcal{C}([0, T] ; Y)} \leq C\left\|\left(\phi^{0}, \phi^{1}, z^{0}, z^{1}\right)\right\|_{Y} .
$$




\subsection{Well-posedness of the controllability problem}

Since we have to deal with nonhomogeneous boundary conditions in system $(2)$, with controls $\left(u_{1}, u_{2}\right) \in$ $L^{2}\left(\Sigma_{c}\right) \times L^{2}(0, T)$ and with initial conditions in $Y$, we define weak solutions by the transposition method. To this end we introduce the adjoint system:

$$
\begin{cases}\psi^{\prime \prime}-c_{f}^{2} \Delta \psi=f & \text { in } Q, \\ \psi=0 & \text { on } \Sigma_{0}, \\ \partial_{n} \psi=w^{\prime} & \text { on } \Sigma_{s}, \\ \psi(T)=0 \quad \text { and } \quad \psi^{\prime}(T)=0 & \text { in } \Omega, \\ w^{\prime \prime}-c_{s}^{2} \Delta_{s} w=-\frac{\rho_{f}}{\rho_{s}} \psi^{\prime}+g & \text { in } \Sigma_{s}, \\ w=0 & \text { on } \sigma_{s}, \\ w(T)=0 \quad \text { and } \quad w^{\prime}(T)=0 & \text { in } \Gamma_{s},\end{cases}
$$

where $(f, g) \in L^{1}\left(0, T ; L^{2}(\Omega)\right) \times L^{1}\left(0, T ; L^{2}\left(\Gamma_{s}\right)\right)$ are given. Notice that, according to the properties of $A$ (see Lemma 2.1) and to Stone's Theorem, the system (4) admits a unique solution $\left(\psi, \psi^{\prime}, w, w^{\prime}\right)$ in $\mathcal{C}([0, T] ; Z)$.

Now we state two existence and uniqueness results, the first one for initial data in $Z$, whereas the second one corresponds to initial data in $Y$.

\subsubsection{Weak solutions of $(2)$}

In the case where $\left(\phi^{0}, \phi^{1}, z^{0}, z^{1}\right) \in Z$, we can define weak solutions of system (2) by the transposition method in a classical way.

Definition 1.5. Let $\left(\phi^{0}, \phi^{1}, z^{0}, z^{1}\right)$ belong to $Z$ and $\left(u_{1}, u_{2}\right)$ belong to $L^{2}\left(\Sigma_{c}\right) \times L^{2}(0, T)$. A pair $(\phi, z) \in$ $L^{\infty}\left(0, T ; L^{2}(\Omega)\right) \times L^{\infty}\left(0, T ; L^{2}\left(\Gamma_{s}\right)\right)$ is called a weak solution of $(2)$ if and only if, for all $(f, g) \in L^{1}\left(0, T ; L^{2}(\Omega)\right) \times$ $L^{1}\left(0, T ; L^{2}\left(\Gamma_{s}\right)\right)$, the following identity holds:

$$
\begin{aligned}
& \int_{Q} f \phi+\alpha \int_{\Sigma_{s}} g z=\int_{\Omega} \phi^{1} \psi(0)-\int_{\Omega} \phi^{0} \psi^{\prime}(0)+\int_{\Gamma_{s}}\left(\alpha z^{1}+c_{f}^{2} \phi^{0}\right) w(0) \\
& -\int_{\Gamma_{s}} z^{0}\left(\alpha w^{\prime}(0)+c_{f}^{2} \psi(0)\right)-c_{f}^{2} \int_{\Sigma_{c}} u_{1} \partial_{n} \psi-\alpha c_{s}^{2} \int_{0}^{T} u_{2} \partial_{x_{1}} w(L)
\end{aligned}
$$

where $(\psi, w)$ is the solution of (4) associated with $(f, g)$.

Theorem 1.6. Let $\left(\phi^{0}, \phi^{1}, z^{0}, z^{1}\right)$ be given in $Z$ and let $\left(u_{1}, u_{2}\right)$ be given in $H_{0}^{2}\left(0, T ; H^{\frac{3}{2}}\left(\Gamma_{c}\right)\right) \times H_{0}^{3}(0, T)$. The system (2) admits a unique weak solution $(\phi, z)$ in $L^{\infty}\left(0, T ; L^{2}(\Omega)\right) \times L^{\infty}\left(0, T ; L^{2}\left(\Gamma_{s}\right)\right)$ in the sense of definition 1.5. Moreover, $\left(\phi, \phi^{\prime}, z, z^{\prime}\right) \in \mathcal{C}\left([0, T] ; H^{1}(\Omega) \times L^{2}(\Omega) \times H^{1}\left(\Gamma_{s}\right) \times L^{2}\left(\Gamma_{s}\right)\right)$ and it satisfies the estimate

$$
\left\|\left(\phi, \phi^{\prime}, z, z^{\prime}\right)\right\|_{\mathcal{C}([0, T] ; Y)} \leq C\left\{\left\|\left(\phi^{0}, \phi^{1}, z^{0}, z^{1}\right)\right\|_{Y}+\left\|u_{1}\right\|_{L^{2}\left(\Sigma_{c}\right)}+\left\|u_{2}\right\|_{L^{2}(0, T)}\right\}
$$

\subsubsection{Very weak solutions of $(2)$}

Let us observe that if $\left(\phi^{0}, \phi^{1}, z^{0}, z^{1}\right) \in Y$, then neither $\left.\phi^{0}\right|_{\Gamma_{s}}$ nor $\alpha z^{1}+\left.c_{f}^{2} \phi^{0}\right|_{\Gamma_{s}}$ are well defined. Thus for initial data in $Y$, we have to introduce some new tools to define weak solutions of equation (2). We first introduce the bounded linear operator $J$ defined in $Z$ by

$$
J\left(\phi^{0}, \phi^{1}, z^{0}, z^{1}\right)=\left(\phi^{0}, \phi^{1}, z^{0}, \alpha z^{1}+\left.c_{f}^{2} \phi^{0}\right|_{\Gamma_{s}}\right) .
$$


In Lemma 3.2, we prove that $J$ can be extended to a bounded linear operator $\tilde{J}$ from $Y$ into $L^{2}(\Omega) \times V^{\prime} \times$ $L^{2}\left(\Gamma_{s}\right) \times H^{-1}\left(\Gamma_{s}\right)$. Thanks to this new operator $\tilde{J}$ we are now able to define weak solutions of equation with initial data in $Y$.

Definition 1.7. Let $\left(\phi^{0}, \phi^{1}, z^{0}, z^{1}\right)$ belong to $Y,\left(u_{1}, u_{2}\right)$ belong to $L^{2}\left(\Sigma_{c}\right) \times L^{2}(0, T)$, and set $\left(\phi^{0}, \phi^{1}, z^{0}, \tilde{z}^{1}\right)=$ $\tilde{J}\left(\phi^{0}, \phi^{1}, z^{0}, z^{1}\right)$. A pair $(\phi, z) \in L^{\infty}\left(0, T ; L^{2}(\Omega)\right) \times L^{\infty}\left(0, T ; L^{2}\left(\Gamma_{s}\right)\right)$ is called a very weak solution of $(2)$ if and only if, for all $(f, g) \in L^{1}\left(0, T ; L^{2}(\Omega)\right) \times L^{1}\left(0, T ; L^{2}\left(\Gamma_{s}\right)\right)$, the following identity holds:

$$
\begin{gathered}
\int_{Q} f \phi+\alpha \int_{\Sigma_{s}} g z=\left\langle\phi^{1}, \psi(0)\right\rangle_{V^{\prime}, V}-\left(\phi^{0}, \psi^{\prime}(0)\right)_{L^{2}(\Omega)}+\left\langle\tilde{z}^{1}, w(0)\right\rangle_{H^{-1}\left(\Gamma_{s}\right), H_{0}^{1}\left(\Gamma_{s}\right)} \\
-\left(z^{0}, \alpha w^{\prime}(0)+c_{f}^{2} \psi(0)\right)_{L^{2}\left(\Gamma_{s}\right)}-c_{f}^{2} \int_{\Sigma_{c}} u_{1} \partial_{n} \psi-\alpha c_{s}^{2} \int_{0}^{T} u_{2} \partial_{x_{1}} w(L),
\end{gathered}
$$

where $(\psi, w)$ is the solution of (4) associated with $(f, g)$.

Remark 1.8. Let us observe that if $\left(\phi^{0}, \phi^{1}, z^{0}, z^{1}\right) \in Z$, then $\tilde{z}^{1}=\alpha z^{1}+\left.c_{f}^{2} \phi^{0}\right|_{\Gamma_{s}}$ and the notion of weak solution in Definition 1.7 coincides with the one of Definition 1.5.

To exhibit some properties of weak solutions of system (2) with initial data in $Y$, we introduce the following system in which the initial data are not specified:

$$
\begin{cases}\psi^{\prime \prime}-c_{f}^{2} \Delta \psi=f & \text { in } Q, \\ \psi=0 & \text { on } \Sigma_{0}, \\ \partial_{n} \psi=w^{\prime} & \text { on } \Sigma_{s} \\ w^{\prime \prime}-c_{s}^{2} \Delta_{s} w=-\frac{\rho_{f}}{\rho_{s}} \psi^{\prime}+g & \text { in } \Sigma_{s}, \\ w=0 & \text { on } \sigma_{s},\end{cases}
$$

where $\left(\psi, \psi^{\prime}, w, w^{\prime}\right) \in \mathcal{C}([0, T] ; Z)$ and $(f, g) \in L^{1}\left(0, T ; L^{2}(\Omega)\right) \times L^{1}\left(0, T ; L^{2}\left(\Gamma_{s}\right)\right)$.

Theorem 1.9. Let $\left(\phi^{0}, \phi^{1}, z^{0}, z^{1}\right)$ be in $Y$ and let $\left(u_{1}, u_{2}\right)$ be in $L^{2}\left(\Sigma_{c}\right) \times L^{2}(0, T)$. The system (2) admits a unique very weak solution $(\phi, z)$ in $L^{\infty}\left(0, T ; L^{2}(\Omega)\right) \times L^{\infty}\left(0, T ; L^{2}\left(\Gamma_{s}\right)\right)$. This solution belongs to $\mathcal{C}\left([0, T] ; L^{2}(\Omega)\right) \cap$ $\mathcal{C}^{1}\left([0, T] ; V^{\prime}\right) \times \mathcal{C}\left([0, T] ; L^{2}\left(\Gamma_{s}\right)\right)$ and satisfies the estimate

$$
\|\phi\|_{L^{\infty}\left(0, T ; L^{2}(\Omega)\right)}+\left\|\phi^{\prime}\right\|_{L^{\infty}\left(0, T ; V^{\prime}\right)}+\|z\|_{L^{\infty}\left(0, T ; L^{2}\left(\Gamma_{s}\right)\right)} \leq C\left\{\left\|\left(\phi^{0}, \phi^{1}, z^{0}, z^{1}\right)\right\|_{Y}+\left\|u_{1}\right\|_{L^{2}\left(\Sigma_{c}\right)}+\left\|u_{2}\right\|_{L^{2}(0, T)}\right\} .
$$

Moreover, setting $\left(\phi^{0}, \phi^{1}, z^{0}, \tilde{z}^{1}\right)=\tilde{J}\left(\phi^{0}, \phi^{1}, z^{0}, z^{1}\right)$, there exists a unique function $\tilde{z} \in \mathcal{C}\left([0, T] ; H^{-1}\left(\Gamma_{s}\right)\right)$ satisfying $\tilde{z}(0)=\tilde{z}^{1}$ and the following formula:

$$
\begin{aligned}
& \int_{0}^{t} \int_{\Omega} f \phi+\alpha \int_{0}^{t} \int_{\Gamma_{s}} g z \\
& =\left\langle\phi^{1}, \psi(0)\right\rangle_{V^{\prime}, V}-\left\langle\phi^{\prime}(t), \psi(t)\right\rangle_{V^{\prime}, V}-\left(\phi^{0}, \psi^{\prime}(0)\right)_{L^{2}(\Omega)}+\left(\phi(t), \psi^{\prime}(t)\right)_{L^{2}(\Omega)} \\
& +\left\langle\tilde{z}^{1}, w(0)\right\rangle_{H^{-1}\left(\Gamma_{s}\right), H_{0}^{1}\left(\Gamma_{s}\right)}-\langle\tilde{z}(t), w(t)\rangle_{H^{-1}\left(\Gamma_{s}\right), H_{0}^{1}\left(\Gamma_{s}\right)}-\left(z^{0}, \alpha w^{\prime}(0)+c_{f}^{2} \psi(0)\right)_{L^{2}\left(\Gamma_{s}\right)} \\
& +\left(z(t), \alpha w^{\prime}(t)+c_{f}^{2} \psi(t)\right)_{L^{2}\left(\Gamma_{s}\right)}-c_{f}^{2} \int_{0}^{t} \int_{\Gamma_{c}} u_{1} \partial_{n} \psi-\alpha c_{s}^{2} \int_{0}^{t} u_{2} \partial_{x_{1}} w(L),
\end{aligned}
$$


for all $t$ in $[0, T]$ and all $\left(\psi, \psi^{\prime}, w, w^{\prime}, f, g\right) \in \mathcal{C}([0, T] ; Z) \times L^{1}\left(0, T ; L^{2}(\Omega)\right) \times L^{1}\left(0, T ; L^{2}\left(\Gamma_{s}\right)\right)$ obeying $(8)$. Besides, there exists $C>0$ independent of $\left(\phi^{0}, \phi^{1}, z^{0}, z^{1}\right)$ such that

$$
\|\tilde{z}\|_{L^{\infty}\left(0, T ; H^{-1}\left(\Gamma_{s}\right)\right)} \leq C\left\{\left\|\left(\phi^{0}, \phi^{1}, z^{0}, z^{1}\right)\right\|_{Y}+\left\|u_{1}\right\|_{L^{2}\left(\Sigma_{c}\right)}+\left\|u_{2}\right\|_{L^{2}(0, T)}\right\} .
$$

Remark 1.10. If $(\phi, z) \in \mathcal{C}([0, T] ; Z)$, then $\tilde{z}=\alpha z^{\prime}+\left.c_{f}^{2} \phi\right|_{\Gamma_{s}}$.

The proof of Theorem 1.9 is based on a generalized direct inequality (see Theorem 5.2 in Appendix).

\subsection{Exact controllability}

We now state the main result of this paper.

Theorem 1.11. There exists $T_{0}>0$ such that, for all $T>T_{0}$, all $\left(\phi^{0}, \phi^{1}, z^{0}, z^{1}\right) \in Y$, there exists $u_{1} \in L^{2}\left(\Sigma_{c}\right)$ and $u_{2} \in L^{2}(0, T)$ for which the solution $(\phi, z)$ of problem (2) obeys:

$$
\phi(T)=\phi^{\prime}(T)=0 \text { in } \Omega, \quad \text { and } \quad z(T)=z^{\prime}(T)=0 \text { in } \Gamma_{s} .
$$

Remark 1.12. Let us underline that Theorem 1.11 gives a null controllability result. Since $\left(e^{t A}\right)_{t \in \mathbb{R}}$ is a group on $Y$, Theorem 1.11 implies the exact controllability in $Y$.

\section{Proof of Theorem 1.2}

The proof of Theorem 1.2 is a direct consequence of the following Lemma.

Lemma 2.1. The operator $(A, D(A))$ is the infinitesimal generator of a strongly continuous unitary group on $Z$.

Proof. To prove the lemma, we show that $(A, D(A))$ is m-dissipative and skew-adjoint in $Z$.

Step 1. $(A, D(A))$ is dissipative in $Z$. For all $\left(\phi_{0}, \phi_{1}, z_{0}, z_{1}\right)$ in $D(A)$, we have:

$$
\begin{aligned}
& \left(A\left(\phi_{0}, \phi_{1}, z_{0}, z_{1}\right),\left(\phi_{0}, \phi_{1}, z_{0}, z_{1}\right)\right)_{Z}=c_{f}^{2} \int_{\Omega} \nabla \phi_{1} \nabla \phi_{0}+c_{f}^{2} \int_{\Omega} \Delta \phi_{0} \phi_{1} \\
& +\alpha c_{s}^{2} \int_{\Gamma_{s}} \nabla_{s} z_{1} \nabla_{s} z_{0}+\alpha c_{s}^{2} \int_{\Gamma_{s}} \Delta_{s} z_{0} z_{1}-\alpha \frac{\rho_{f}}{\rho_{s}} \int_{\Gamma_{s}} \phi_{1} z_{1}=0 .
\end{aligned}
$$

Step 2. $(A, D(A))$ is m-dissipative. Let $F=\left(f_{1}, f_{2}, f_{3}, f_{4}\right)$ be fixed in $Z$. We want to solve:

$$
U=\left(\phi_{0}, \phi_{1}, z_{0}, z_{1}\right) \in D(A), \quad U-A U=F .
$$

This is clearly equivalent to

$$
\begin{cases}\phi_{0} \in V, \Delta \phi_{0} \in L^{2}(\Omega), & \phi_{0}-c_{f}^{2} \Delta \phi_{0}=f_{1}+f_{2} \text { in } \Omega \\ \phi_{1} \in V, & \phi_{1}=\phi_{0}-f_{1} \text { in } \Omega \\ z_{0} \in H^{2}\left(\Gamma_{s}\right) \cap H_{0}^{1}\left(\Gamma_{s}\right), & z_{0}-c_{s}^{2} \Delta_{s} z_{0}+\frac{\rho_{f}}{\rho_{s}} \phi_{0}=\frac{\rho_{f}}{\rho_{s}} f_{1}+f_{3}+f_{4} \text { on } \Gamma_{s} \\ z_{1} \in H_{0}^{1}\left(\Gamma_{s}\right), & z_{1}=z_{0}-f_{3}=\partial_{n} \phi_{0} \text { on } \Gamma_{s}\end{cases}
$$

If we set

$$
g_{1}:=f_{1}+f_{2} \in L^{2}(\Omega), \quad \text { and } \quad g_{2}:=\frac{\rho_{f}}{\rho_{s}} f_{1}+f_{3}+f_{4} \in L^{2}\left(\Gamma_{s}\right),
$$


the system satisfied by $\left(\phi_{0}, z_{0}\right)$ may be rewritten in the form

$$
\begin{cases}\phi_{0} \in V, \Delta \phi_{0} \in L^{2}(\Omega), & \phi_{0}-c_{f}^{2} \Delta \phi_{0}=g_{1} \text { in } \Omega, \quad \partial_{n} \phi_{0}=z_{0}-f_{3} \text { on } \Gamma_{s}, \\ z_{0} \in H^{2}\left(\Gamma_{s}\right) \cap H_{0}^{1}\left(\Gamma_{s}\right), & z_{0}-c_{s}^{2} \Delta_{s} z_{0}+\frac{\rho_{f}}{\rho_{s}} \phi_{0}=g_{2} \text { on } \Gamma_{s} .\end{cases}
$$

Let us equip $W=V \times H_{0}^{1}\left(\Gamma_{s}\right)$ with the following inner product:

$$
\left(\left(\phi_{0}, z_{0}\right),\left(\hat{\phi}_{0}, \hat{z}_{0}\right)\right)_{W}=\int_{\Omega} \phi_{0} \hat{\phi}_{0}+c_{f}^{2} \int_{\Omega} \nabla \phi_{0} \nabla \hat{\phi}_{0}+\alpha \int_{\Gamma_{s}} z_{0} \hat{z}_{0}+\alpha c_{s}^{2} \int_{\Gamma_{s}} \nabla_{s} z_{0} \nabla_{s} \hat{z}_{0} .
$$

We define the bilinear form $a$ on $W$ by:

$$
a\left(\left(\phi_{0}, z_{0}\right),\left(\hat{\phi}_{0}, \hat{z}_{0}\right)\right)=\int_{\Omega} \phi_{0} \hat{\phi}_{0}+c_{f}^{2} \int_{\Omega} \nabla \phi_{0} \nabla \hat{\phi}_{0}+\alpha \int_{\Gamma_{s}} z_{0} \hat{z}_{0}+\alpha c_{s}^{2} \int_{\Gamma_{s}} \nabla_{s} z_{0} \nabla_{s} \hat{z}_{0}+c_{f}^{2} \int_{\Gamma_{s}}\left(\phi_{0} \hat{z}_{0}-z_{0} \hat{\phi}_{0}\right),
$$

and the linear form $L$ on $W$ by:

$$
L\left(\hat{\phi}_{0}, \hat{z}_{0}\right)=\int_{\Omega} g_{1} \hat{\phi}_{0}+\alpha \int_{\Gamma_{s}} g_{2} \hat{z}_{0}-c_{f}^{2} \int_{\Gamma_{s}} f_{3} \hat{\phi}_{0}
$$

The existence of a unique weak solution $\left(\phi_{0}, z_{0}\right) \in W$ to the system (11) follows from the Lax Milgram Lemma applied to the variational problem:

$$
\text { find }\left(\phi_{0}, z_{0}\right) \in W \quad \text { such that } a\left(\left(\phi_{0}, z_{0}\right),\left(\hat{\phi}_{0}, \hat{z}_{0}\right)\right)=L\left(\hat{\phi}_{0}, \hat{z}_{0}\right), \quad \text { for all }\left(\hat{\phi}_{0}, \hat{z}_{0}\right) \in W \text {. }
$$

Next we can easily show that $\Delta \phi_{0} \in L^{2}(\Omega)$ and $z_{0} \in H^{2}\left(\Gamma_{s}\right) \cap H_{0}^{1}\left(\Gamma_{s}\right)$. We have $\phi_{1}=\phi_{0}-f_{1} \in V$ and $z_{1}=z_{0}-f_{3} \in H_{0}^{1}\left(\Gamma_{s}\right)$. Therefore, there exists $U=\left(\phi_{0}, \phi_{1}, z_{0}, z_{1}\right) \in D(A)$ such that $U-A U=F$, and $A$ is m-dissipative on $Z$.

Step 3. $A$ is skewadjoint. We can easily check that the adjoint of $A$ with respect to the pivot space $Z$ is defined by

The proof is complete.

$$
D\left(A^{*}\right)=D(A) \quad \text { and } \quad A^{*}=-A .
$$

\section{Proof of Theorems 1.6 AND 1.9}

\subsection{Preliminary lemmas}

In order to prove Theorems 1.6 and 1.9, we will use some lemmas, which simplify the computations. First, we introduce the system

$$
\begin{cases}\psi^{\prime \prime}-c_{f}^{2} \Delta \psi=f & \text { in } Q, \\ \psi=0 & \text { on } \Sigma_{0}, \\ \partial_{n} \psi=w^{\prime} & \text { on } \Sigma_{s}, \\ \psi(\tau)=\psi_{\tau} \quad \text { and } \quad \psi^{\prime}(\tau)=0 & \text { in } \Omega, \\ w^{\prime \prime}-c_{s}^{2} \Delta_{s} w=-\frac{\rho_{f}}{\rho_{s}} \psi^{\prime}+g & \text { in } \Sigma_{s}, \\ w=0 & \text { on } \sigma_{s}, \\ w(\tau)=w_{\tau} \quad \text { and } \quad w^{\prime}(\tau)=0 & \text { in } \Gamma_{s},\end{cases}
$$

where $\tau \in[0, T],\left(\psi_{\tau}, w_{\tau}\right) \in V \times H_{0}^{1}\left(\Gamma_{s}\right)$ and $(f, g) \in L^{1}\left(0, T ; L^{2}(\Omega)\right) \times L^{1}\left(0, T ; L^{2}\left(\Gamma_{s}\right)\right)$. 
Lemma 3.1. Let $\tau$ be in $[0, T],\left(\psi_{\tau}, w_{\tau}\right)$ be in $V \times H_{0}^{1}\left(\Gamma_{s}\right)$, and let $(f, g)$ be in $L^{1}\left(0, T ; L^{2}(\Omega)\right) \times L^{1}\left(0, T ; L^{2}\left(\Gamma_{s}\right)\right)$. The system (13) admits a unique weak solution $\left(\psi, \psi^{\prime}, w, w^{\prime}\right)$ in $\mathcal{C}([0, T] ; Z)$, satisfying the following estimate:

$$
\begin{aligned}
& \left\|\left(\psi, \psi^{\prime}, w, w^{\prime}\right)\right\|_{\mathcal{C}([0, T] ; Z)}^{2}+c_{f}^{2}\left\|\partial_{n} \psi\right\|_{L^{2}\left(\Sigma_{c}\right)}^{2}+\alpha c_{s}^{2}\left\|\partial_{x_{1}} w(L)\right\|_{L^{2}(0, T)}^{2} \\
& \leq C_{T}\left\{\left\|\psi_{\tau}\right\|_{V}^{2}+\left\|w_{\tau}\right\|_{H_{0}^{1}\left(\Gamma_{s}\right)}^{2}+\|f\|_{L^{1}\left(0, T ; L^{2}(\Omega)\right)}^{2}+\alpha\|g\|_{L^{1}\left(0, T ; L^{2}\left(\Gamma_{s}\right)\right)}^{2}\right\}
\end{aligned}
$$

where $C_{T}>0$ depends on $T$, but is independent of $\tau,\left(\psi_{\tau}, w_{\tau}\right)$ and of $(f, g)$.

Proof. Due to Lemma 2.1 and to classical results for evolution equations, the weak solution of system (13) obeys the estimate

$$
\left\|\left(\psi, \psi^{\prime}, w, w^{\prime}\right)\right\|_{\mathcal{C}([0, T] ; Z)}^{2} \leq K\left\{\left\|\psi_{\tau}\right\|_{V}^{2}+\left\|w_{\tau}\right\|_{H_{0}^{1}\left(\Gamma_{s}\right)}^{2}+\|f\|_{L^{1}\left(0, T ; L^{2}(\Omega)\right)}^{2}+\alpha\|g\|_{L^{1}\left(0, T ; L^{2}\left(\Gamma_{s}\right)\right)}^{2}\right\}
$$

where the constant $K$ is independent of $\tau$. ¿From Theorem 5.2 in Appendix we deduce that:

$c_{f}^{2} \int_{\Sigma_{c}}\left|\partial_{n} \psi\right|^{2}+\alpha c_{s}^{2} \int_{0}^{T}\left|\partial_{x_{1}} w(L)\right|^{2} \leq(1+K) C_{T}\left\{\left\|\psi_{\tau}\right\|_{V}^{2}+\left\|w_{\tau}\right\|_{H_{0}^{1}\left(\Gamma_{s}\right)}^{2}+\|f\|_{L^{1}\left(0, T ; L^{2}(\Omega)\right)}^{2}+\alpha\|g\|_{L^{1}\left(0, T ; L^{2}\left(\Gamma_{s}\right)\right)}^{2}\right\}$.

Since $K$ is independent of $\tau$, taking $C=(1+K) C_{T}$, the proof is complete.

Recall that $J$ is defined in $Z$ by

$$
J\left(\phi^{0}, \phi^{1}, z^{0}, z^{1}\right)=\left(\phi^{0}, \phi^{1}, z^{0}, \alpha z^{1}+\left.c_{f}^{2} \phi^{0}\right|_{\Gamma_{s}}\right) .
$$

Lemma 3.2. $J$ is an isomorphism from $Z$ to $Z$. It may be uniquely extended to a bounded linear operator $\tilde{J}$ from $Y$ to $L^{2}(\Omega) \times V^{\prime} \times L^{2}\left(\Gamma_{s}\right) \times H^{-1}\left(\Gamma_{s}\right)$. Moreover $\tilde{J}$ is an isomorphism from $Y$ to $L^{2}(\Omega) \times V^{\prime} \times L^{2}\left(\Gamma_{s}\right) \times H^{-1}\left(\Gamma_{s}\right)$.

Proof. It is obvious that $J$ is an isomorphism from $Z$ to $Z$. Observe that $J$ is also continuous from $\left(Z,\|\cdot\|_{Y}\right)$ to $L^{2}(\Omega) \times V^{\prime} \times L^{2}\left(\Gamma_{s}\right) \times H^{-1}\left(\Gamma_{s}\right)$, since we have

$$
\left\|J\left(\phi^{0}, \phi^{1}, z^{0}, z^{1}\right)\right\|_{L^{2}(\Omega) \times V^{\prime} \times L^{2}\left(\Gamma_{s}\right) \times H^{-1}\left(\Gamma_{s}\right)}^{2}=\left\|\left(\phi^{0}, \phi^{1}, z^{0}, z^{1}\right)\right\|_{Y}^{2} .
$$

Thus, $J$ can be uniquely extended to a bounded linear operator $\tilde{J}$ from $Y$ to $L^{2}(\Omega) \times V^{\prime} \times L^{2}\left(\Gamma_{s}\right) \times H^{-1}\left(\Gamma_{s}\right)$. Moreover, since

$$
\left\|\tilde{J}\left(\phi^{0}, \phi^{1}, z^{0}, z^{1}\right)\right\|_{L^{2}(\Omega) \times V^{\prime} \times L^{2}\left(\Gamma_{s}\right) \times H^{-1}\left(\Gamma_{s}\right)}^{2}=\left\|\left(\phi^{0}, \phi^{1}, z^{0}, z^{1}\right)\right\|_{Y}^{2},
$$

$\tilde{J}$ is an isometry from $Y$ to $L^{2}(\Omega) \times V^{\prime} \times L^{2}\left(\Gamma_{s}\right) \times H^{-1}\left(\Gamma_{s}\right)$, and in particular, $\tilde{J}$ is an isomorphism from $Y$ to $L^{2}(\Omega) \times V^{\prime} \times L^{2}\left(\Gamma_{s}\right) \times H^{-1}\left(\Gamma_{s}\right)$. Lemma 3.2 is proved.

\subsection{Proof of Theorem 1.6}

Step 1. Existence of a solution $(\phi, z)$ in $L^{\infty}\left(0, T ; L^{2}(\Omega)\right) \times L^{\infty}\left(0, T ; L^{2}\left(\Gamma_{s}\right)\right)$. Let $\left(\phi^{0}, \phi^{1}, z^{0}, z^{1}\right)$ be in $Z$ and $\left(u_{1}, u_{2}\right)$ be in $H_{0}^{2}\left(0, T ; H^{\frac{3}{2}}\left(\Gamma_{c}\right)\right) \times H_{0}^{3}(0, T)$. Let $\mathcal{L}$ be the linear form on $L^{2}(Q) \times L^{2}\left(\Sigma_{s}\right)$, defined by:

$$
\begin{aligned}
& \mathcal{L}(f, g)=\int_{\Omega} \phi^{1} \psi(0)-\int_{\Omega} \phi^{0} \psi^{\prime}(0)+\int_{\Gamma_{s}}\left(\alpha z^{1}+c_{f}^{2} \phi^{0}\right) w(0) \\
& -\int_{\Gamma_{s}} z_{0}\left(\alpha w^{\prime}(0)+c_{f}^{2} \psi(0)\right)-c_{f}^{2} \int_{\Sigma_{c}} u_{1} \partial_{n} \psi-\alpha c_{s}^{2} \int_{0}^{T} u_{2} \partial_{x_{1}} w(L),
\end{aligned}
$$


where $(\psi, w)$ is the solution of $(4)$ corresponding to $(f, g) \in L^{2}(Q) \times L^{2}\left(\Sigma_{s}\right)$. Let us show that $\mathcal{L}$ is continuous in $L^{2}(Q) \times L^{2}\left(\Sigma_{s}\right)$. With Lemma 3.1, we have

$$
\begin{aligned}
& \begin{aligned}
&|\mathcal{L}(f, g)| \leq C\left(\left\|\left(\phi_{0}, \phi_{1}, z_{0}, z_{1}\right)\right\|_{Y}+\left\|u_{1}\right\|_{L^{2}\left(\Sigma_{c}\right)}+\left\|u_{2}\right\|_{L^{2}(0, T)}\right) \\
& \times\left(\left\|\left(\psi, \psi^{\prime}, w, w^{\prime}\right)\right\|_{\mathcal{C}([0, T] ; Z)}+c_{f}^{2}\left\|\partial_{n} \psi\right\|_{L^{2}\left(\Sigma_{c}\right)}+\alpha c_{s}^{2}\left\|\partial_{x_{1}} w(L)\right\|_{L^{2}(0, T)}\right) \\
& \leq C\left\{\|f\|_{L^{2}(Q)}+\|g\|_{L^{2}\left(\Sigma_{s}\right)}\right\} .
\end{aligned}
\end{aligned}
$$

Thus $\mathcal{L}$ is continuous in $L^{2}(Q) \times L^{2}\left(\Sigma_{s}\right)$ endowed with the inner product

$$
((f, g),(\phi, z)) \longmapsto \int_{Q} f \phi+\alpha \int_{\Sigma_{s}} g z .
$$

¿From Riesz' Theorem we deduce that there exists a unique pair $(\phi, z)$ belonging to $L^{2}(Q) \times L^{2}\left(\Sigma_{s}\right)$ such that

$$
\mathcal{L}(f, g)=\int_{Q} f \phi+\alpha \int_{\Sigma_{s}} g z
$$

for all $(f, g) \in L^{2}(Q) \times L^{2}\left(\Sigma_{s}\right)$. Thus the pair $(\phi, z)$ satisfies identity (5). Now we have to prove that $(\phi, z)$ belongs to $L^{\infty}\left(0, T ; L^{2}(\Omega)\right) \times L^{\infty}\left(0, T ; L^{2}\left(\Gamma_{s}\right)\right)$.

Taking $g=0$ in formula (5) and using Lemma 3.1, we obtain:

$$
\begin{aligned}
& \left|\int_{Q} f \phi\right| \leq\left\|\left(\phi^{0}, \phi^{1}, z^{0}, z^{1}\right)\right\|_{Y}\left\|\left(\psi, \psi^{\prime}, w, w^{\prime}\right)\right\|_{\mathcal{C}([0, T] ; Z)} \\
& +c_{f}^{2}\left\|u_{1}\right\|_{L^{2}\left(\Sigma_{c}\right)}\left\|\partial_{n} \psi\right\|_{L^{2}\left(\Sigma_{c}\right)}+\alpha c_{s}^{2}\left\|u_{2}\right\|_{L^{2}(0, T)}\left\|\partial_{x_{1}} w(L)\right\|_{L^{2}(0, T)} \\
& \leq C\|f\|_{L^{1}\left(0, T ; L^{2}(\Omega)\right)}\left\{\left\|\left(\phi^{0}, \phi^{1}, z^{0}, z^{1}\right)\right\|_{Y}+\left\|u_{1}\right\|_{L^{2}\left(\Sigma_{c}\right)}+\left\|u_{2}\right\|_{L^{2}(0, T)}\right\} .
\end{aligned}
$$

Therefore, it yields:

$$
\|\phi\|_{L^{\infty}\left(0, T ; L^{2}(\Omega)\right)}=\sup \left\{\left|\int_{Q} f \phi\right|,\|f\|_{L^{1}\left(0, T ; L^{2}(\Omega)\right)}=1\right\} \leq C\left\{\left\|\left(\phi^{0}, \phi^{1}, z^{0}, z^{1}\right)\right\|_{Y}+\left\|u_{1}\right\|_{L^{2}\left(\Sigma_{c}\right)}+\left\|u_{2}\right\|_{L^{2}(0, T)}\right\} .
$$

A similar computation shows that:

$$
\|z\|_{L^{\infty}\left(0, T ; L^{2}\left(\Gamma_{s}\right)\right)} \leq C\left\{\left\|\left(\phi^{0}, \phi^{1}, z^{0}, z^{1}\right)\right\|_{Y}+\left\|u_{1}\right\|_{L^{2}\left(\Sigma_{c}\right)}+\left\|u_{2}\right\|_{L^{2}(0, T)}\right\} .
$$

Therefore $(\phi, z) \in L^{\infty}\left(0, T ; L^{2}(\Omega)\right) \times L^{\infty}\left(0, T ; L^{2}\left(\Gamma_{s}\right)\right)$ is a weak solution of system (2).

Step 2. Regularity of $(\phi, z)$. Let $c \in H^{3}\left(0, T ; H^{\frac{3}{2}}\left(\Gamma_{s}\right)\right)$ be such that

$$
\Delta c=0 \text { on } \Sigma_{s}, \quad c(0, t)=0 \text { and } c(L, t)=u_{2}(t) \text { in }(0, T) .
$$

(Actually $c(x, t)=x u_{2}(t) / L$ since $\Gamma_{s}$ is a one-dimensional domain). Since $u_{1} \in H_{0}^{2}\left(0, T ; H^{3 / 2}(\Omega)\right)$ and $c^{\prime} \in$ $H^{2}\left(0, T ; L^{2}\left(\Gamma_{s}\right)\right)$, there exists $\zeta \in H^{2}\left(0, T ; H^{3 / 2-\varepsilon}(\Omega)\right)$ for all $\varepsilon>0$, such that

$$
-\Delta \zeta=0 \text { in } Q, \quad \zeta=u_{1} \text { on } \Sigma_{c}, \quad \zeta=0 \text { on } \Sigma_{0} \backslash \Sigma_{c} \text { and } \partial_{n} \zeta=c^{\prime} \text { on } \Sigma_{s} .
$$


(See for example [11].) Let us set $(\Phi, \mathcal{Z})=(\phi-\zeta, z-c)$. Then, $(\Phi, \mathcal{Z})$ is solution of the following system:

$$
\begin{cases}\Phi^{\prime \prime}-c_{f}^{2} \Delta \Phi=-\zeta^{\prime \prime} & \text { in } Q \\ \Phi=0 & \text { on } \Sigma_{0} \\ \partial_{n} \Phi=\mathcal{Z}^{\prime} & \text { on } \Sigma_{s} \\ \Phi(0)=\phi^{0}-\zeta(0) \quad \text { and } \quad \Phi^{\prime}(0)=\phi^{1}-\zeta^{\prime}(0) & \text { in } \Omega \\ \mathcal{Z}^{\prime \prime}-c_{s}^{2} \Delta_{s} \mathcal{Z}=-\frac{\rho_{f}}{\rho_{s}} \Phi^{\prime}-\frac{\rho_{f}}{\rho_{s}} \zeta^{\prime}-c^{\prime \prime} & \text { in } \Sigma_{s} \\ \mathcal{Z}=0 & \text { on } \sigma_{s} \\ \mathcal{Z}(0)=z^{0}-c(0) \quad \text { and } \quad \mathcal{Z}^{\prime}(0)=z^{1}-c^{\prime}(0) & \text { in } \Gamma_{s}\end{cases}
$$

Hence, setting

$$
C=\left(\begin{array}{c}
0 \\
-\zeta^{\prime \prime} \\
0 \\
-\frac{\rho_{f}}{\rho_{s}} \zeta^{\prime}-c^{\prime \prime}
\end{array}\right), \quad U=\left(\begin{array}{c}
\Phi \\
\Phi^{\prime} \\
\mathcal{Z} \\
\mathcal{Z}^{\prime}
\end{array}\right) \text { and } U_{0}=\left(\begin{array}{c}
\phi^{0}-\zeta(0) \\
\phi^{1}-\zeta^{\prime}(0) \\
z^{0}-c(0) \\
z^{1}-c^{\prime}(0)
\end{array}\right)
$$

the system (16) can be written in the form:

$$
\frac{d U}{d t}=A U+C, \quad U(0)=U_{0}
$$

¿From the regularity of $(\zeta, c)$ we deduce that $\left(C, U_{0}\right) \in L^{2}(0, T ; Z) \times Z$. Due to Lemma 2.1 , the system (16) has a unique solution $\left(\Phi, \Phi^{\prime}, \mathcal{Z}, \mathcal{Z}^{\prime}\right) \in \mathcal{C}([0, T] ; Z)$, and therefore

$$
\phi=\Phi+\zeta \in \mathcal{C}\left([0, T] ; H^{1}(\Omega)\right) \cap \mathcal{C}^{1}\left([0, T] ; L^{2}(\Omega)\right), \quad z=\mathcal{Z}+c \in \mathcal{C}\left([0, T] ; H^{1}\left(\Gamma_{s}\right)\right) \cap \mathcal{C}^{1}\left([0, T] ; L^{2}\left(\Gamma_{s}\right)\right)
$$

Step 3. Estimate of $\left\|\phi^{\prime}\right\|_{L^{\infty}\left(0, T ; V^{\prime}\right)}$. Fix $\left.\left.\tau \in\right] 0, T\right]$. Let $(\psi, w)$ be the solution of (13) corresponding to $\psi_{\tau} \in V, w_{\tau}=0$ and $(f, g)=(0,0)$. An easy computation shows that:

$$
\begin{aligned}
& \int_{\Omega} \phi^{\prime}(\tau) \psi_{\tau}=\int_{\Omega} \phi^{1} \psi(0)-\int_{\Omega} \phi^{0} \psi^{\prime}(0)+\int_{\Gamma_{s}}\left(\alpha z^{1}+c_{f}^{2} \phi^{0}\right) w(0) \\
& -\int_{\Gamma_{s}} z^{0}\left(\alpha w^{\prime}(0)+c_{f}^{2} \psi(0)\right)+c_{f}^{2} \int_{\Gamma_{s}} z(\tau) \psi_{\tau}-c_{f}^{2} \int_{0}^{\tau} \int_{\Gamma_{c}} u_{1} \partial_{n} \psi-\alpha c_{s}^{2} \int_{0}^{\tau} u_{2} \partial_{x_{1}} w(L) .
\end{aligned}
$$

Due to Lemma 3.1, there exists $C>0$, independent of $\tau$, such that:

$$
\begin{aligned}
\left|\int_{\Omega} \phi^{\prime}(\tau) \psi_{\tau}\right| \leq & \left\|\left(\phi^{0}, \phi^{1}, z^{0}, z^{1}\right)\right\|_{Y}\left\|\left(\psi, \psi^{\prime}, w, w^{\prime}\right)\right\|_{\mathcal{C}([0, T] ; Z)}+\left|c_{f}^{2} \int_{\Gamma_{s}} z(\tau) \psi_{\tau}\right| \\
& +c_{f}^{2}\left\|u_{1}\right\|_{L^{2}\left(\Sigma_{c}\right)}\left\|\partial_{n} \psi\right\|_{L^{2}\left(\Sigma_{c}\right)}+\alpha c_{s}^{2}\left\|u_{2}\right\|_{L^{2}(0, T)}\left\|\partial_{x_{1}} w(L)\right\|_{L^{2}(0, T)} \\
\leq & C\left\|\psi_{\tau}\right\|_{V}\left(\|\left(\phi^{0}, \phi^{1}, z^{0}, z^{1}\left\|_{Y}+\right\| u_{1}\left\|_{L^{2}\left(\Sigma_{c}\right)}+\right\| u_{2} \|_{L^{2}(0, T)}\right)+\left|c_{f}^{2} \int_{\Gamma_{s}} z(\tau) \psi_{\tau}\right|\right.
\end{aligned}
$$

Moreover, due to the estimate of $\|z\|_{L^{\infty}\left(0, T ; L^{2}\left(\Gamma_{s}\right)\right)}$ in Step 1, we have

$$
\left|\int_{\Gamma_{s}} z(\tau) \psi_{\tau}\right| \leq C\left\|\psi_{\tau}\right\|_{V}\left\{\left\|\left(\phi^{0}, \phi^{1}, z^{0}, z^{1}\right)\right\|_{Y}+\left\|u_{1}\right\|_{L^{2}\left(\Sigma_{c}\right)}+\left\|u_{2}\right\|_{L^{2}(0, T)}\right\} .
$$


Therefore, with the previous estimate we obtain

$$
\left|\int_{\Omega} \phi^{\prime}(\tau) \psi_{\tau}\right| \leq C\left\|\psi_{\tau}\right\|_{V}\left\{\left\|\left(\phi^{0}, \phi^{1}, z^{0}, z^{1}\right)\right\|_{Y}+\left\|u_{1}\right\|_{L^{2}\left(\Sigma_{c}\right)}+\left\|u_{2}\right\|_{L^{2}(0, T)}\right\}
$$

where $C>0$ is independent of $\tau$. It follows that

$$
\left\|\phi^{\prime}(\tau)\right\|_{V^{\prime}}=\sup \left\{\left|\int_{\Omega} \phi^{\prime}(\tau) \psi_{\tau}\right|,\left\|\psi_{\tau}\right\|_{V}=1\right\} \leq C\left\{\left\|\left(\phi^{0}, \phi^{1}, z^{0}, z^{1}\right)\right\|_{Y}+\left\|u_{1}\right\|_{L^{2}\left(\Sigma_{c}\right)}+\left\|u_{2}\right\|_{L^{2}(0, T)}\right\} .
$$

Since the constant $C$ is independent of $\tau$, we obtain:

$$
\left\|\phi^{\prime}\right\|_{L^{\infty}\left(0, T ; V^{\prime}\right)} \leq C\left\{\left\|\left(\phi^{0}, \phi^{1}, z^{0}, z^{1}\right)\right\|_{Y}+\left\|u_{1}\right\|_{L^{2}\left(\Sigma_{c}\right)}+\left\|u_{2}\right\|_{L^{2}(0, T)}\right\} .
$$

Step 4. Estimate of $\left\|\alpha z^{\prime}+c_{f}^{2} \phi\right\|_{L^{\infty}\left(0, T ; H^{-1}\left(\Gamma_{s}\right)\right)}$. Fix $\left.\left.\tau \in\right] 0, T\right]$. Let $(\psi, w)$ be the solution of (13) corresponding to $w_{\tau} \in H_{0}^{1}\left(\Gamma_{s}\right), \psi_{\tau}=0$ and $(f, g)=(0,0)$. An easy computation shows that:

$$
\begin{aligned}
& \int_{\Gamma_{s}}\left(\alpha z^{\prime}(\tau)+c_{f}^{2} \phi(\tau)\right) w_{\tau}=\int_{\Omega} \phi^{1} \psi(0)-\int_{\Omega} \phi^{0} \psi^{\prime}(0)+\int_{\Gamma_{s}}\left(\alpha z^{1}+c_{f}^{2} \phi^{0}\right) w(0) \\
& -\int_{\Gamma_{s}} z^{0}\left(\alpha w^{\prime}(0)+c_{f}^{2} \psi(0)\right)-c_{f}^{2} \int_{0}^{\tau} \int_{\Gamma_{c}} u_{1} \partial_{n} \psi-\alpha c_{s}^{2} \int_{0}^{\tau} u_{2} \partial_{x_{1}} w(L) .
\end{aligned}
$$

Therefore, we have:

$$
\begin{aligned}
& \left|\int_{\Gamma_{s}}\left(\alpha z^{\prime}(\tau)+c_{f}^{2} \phi(\tau)\right) w_{\tau}\right| \leq\left\|\left(\phi^{0}, \phi^{1}, z^{0}, z^{1}\right)\right\|_{Y}\left\|\left(\psi, \psi^{\prime}, w, w^{\prime}\right)\right\|_{\mathcal{C}([0, T] ; Z)} \\
& +c_{f}^{2}\left\|u_{1}\right\|_{L^{2}\left(\Sigma_{c}\right)}\left\|\partial_{n} \psi\right\|_{L^{2}\left(\Sigma_{c}\right)}+\alpha c_{s}^{2}\left\|u_{2}\right\|_{L^{2}(0, T)}\left\|\partial_{x_{1}} w(L)\right\|_{L^{2}(0, T)} .
\end{aligned}
$$

Due to Lemma 3.1, there exists $C>0$, independent of $\tau$, such that:

$$
\left|\int_{\Gamma_{s}}\left(\alpha z^{\prime}(\tau)+c_{f}^{2} \phi(\tau)\right) w_{\tau}\right| \leq C\left\|w_{\tau}\right\|_{H_{0}^{1}\left(\Gamma_{s}\right)}\left\{\left\|\left(\phi^{0}, \phi^{1}, z^{0}, z^{1}\right)\right\|_{Y}+\left\|u_{1}\right\|_{L^{2}\left(\Sigma_{c}\right)}+\left\|u_{2}\right\|_{L^{2}(0, T)}\right\} .
$$

As in step 3, we obtain:

$$
\left\|\alpha z^{\prime}+c_{f}^{2} \phi\right\|_{L^{\infty}\left(0, T ; H^{-1}\left(\Gamma_{s}\right)\right)} \leq C\left\{\left\|\left(\phi^{0}, \phi^{1}, z^{0}, z^{1}\right)\right\|_{Y}+\left\|u_{1}\right\|_{L^{2}\left(\Sigma_{c}\right)}+\left\|u_{2}\right\|_{L^{2}(0, T)}\right\} .
$$

The proof of Theorem 1.6 is complete.

\subsection{Proof of Theorem 1.9}

Let $\left(\phi^{0}, \phi^{1}, z^{0}, z^{1}\right)$ belong to $Y$, and $\left(u_{1}, u_{2}\right)$ belong to $L^{2}\left(\Sigma_{c}\right) \times L^{2}(0, T)$. Set $\left(\phi^{0}, \phi^{1}, z^{0}, \tilde{z}^{1}\right)=\tilde{J}\left(\phi^{0}, \phi^{1}, z^{0}, z^{1}\right)$. To prove the existence of a weak solution of system (2), we proceed by approximation. There exist sequences $\left(\left(\phi_{n}^{0}, \phi_{n}^{1}, z_{n}^{0}, z_{n}^{1}\right)\right)_{n} \subset Z$ and $\left(\left(u_{n}^{1}, u_{n}^{2}\right)\right)_{n} \subset H^{2}\left(0, T ; H^{\frac{3}{2}}\left(\Gamma_{c}\right)\right) \times H_{0}^{3}(0, T)$ such that

$$
\left\{\begin{array}{l}
\left(\phi_{n}^{0}, \phi_{n}^{1}, z_{n}^{0}\right) \rightarrow\left(\phi^{0}, \phi^{1}, z^{0}\right) \text { in } L^{2}(\Omega) \times V^{\prime} \times L^{2}\left(\Gamma_{s}\right), \\
\alpha z_{n}^{1}+c_{f}^{2} \phi_{n}^{0} \rightarrow \tilde{z}^{1} \text { in } H^{-1}\left(\Gamma_{s}\right), \\
\left(u_{n}^{1}, u_{n}^{2}\right) \rightarrow\left(u_{1}, u_{2}\right) \quad \text { in } L^{2}\left(\Sigma_{c}\right) \times L^{2}(0, T) .
\end{array}\right.
$$


Then, denoting by $\left(\phi_{n}, z_{n}\right)$ the solution of (2) associated with $\left(\phi_{n}^{0}, \phi_{n}^{1}, z_{n}^{0}, z_{n}^{1}, u_{n}^{1}, u_{n}^{2}\right)$, from Theorem 1.6 , it follows that:

$$
\left(\phi_{n}, \phi_{n}^{\prime}, z_{n}, z_{n}^{\prime}\right) \quad \rightarrow \quad\left(\phi, \phi^{\prime}, z, z^{\prime}\right) \quad \text { in } \quad \mathcal{C}([0, T] ; Y)
$$

for some $\left(\phi, \phi^{\prime}, z, z^{\prime}\right) \in \mathcal{C}([0, T] ; Y)$, and $\left(\phi, \phi^{\prime}, z, z^{\prime}\right)$ satisfies estimate $(6)$. Moreover $\left(\alpha z_{n}^{\prime}+c_{f}^{2} \phi_{n}\right)_{n}$ is a Cauchy sequence in $\mathcal{C}\left([0, T] ; H^{-1}\left(\Gamma_{s}\right)\right)$, and it converges in $\mathcal{C}\left([0, T] ; H^{-1}\left(\Gamma_{s}\right)\right)$ to some $\tilde{z} \in \mathcal{C}\left([0, T] ; H^{-1}\left(\Gamma_{s}\right)\right)$. In particular, we have $\tilde{z}(0)=\tilde{z}^{1}$. Let $(\psi, w)$ be the solution of $(4)$ corresponding to $(f, g) \in L^{1}\left(0, T ; L^{2}(\Omega)\right) \times L^{1}\left(0, T ; L^{2}\left(\Gamma_{s}\right)\right)$. Let us recall that the pair $\left(\phi_{n}, z_{n}\right)$ obeys (see Definition 1.7):

$$
\begin{aligned}
& \int_{Q} f \phi_{n}+\alpha \int_{\Sigma_{s}} g z_{n} \\
& =\left\langle\phi_{n}^{1}, \psi(0)\right\rangle_{V^{\prime}, V}-\left(\phi_{n}^{0}, \psi^{\prime}(0)\right)_{L^{2}(\Omega)}+\left\langle\alpha z_{n}^{1}+c_{f}^{2} \phi_{n}^{0}, w(0)\right\rangle_{H^{-1}\left(\Gamma_{s}\right), H_{0}^{1}\left(\Gamma_{s}\right)} \\
& -\left(z_{n}^{0}, \alpha w^{\prime}(0)+c_{f}^{2} \psi(0)\right)_{L^{2}\left(\Gamma_{s}\right)}-c_{f}^{2} \int_{\Sigma_{c}} u_{n}^{1} \partial_{n} \psi-\alpha c_{s}^{2} \int_{0}^{T} u_{n}^{2} \partial_{x_{1}} w(L),
\end{aligned}
$$

for all $(f, g) \in L^{1}\left(0, T ; L^{2}(\Omega)\right) \times L^{1}\left(0, T ; L^{2}\left(\Gamma_{s}\right)\right)$, where $(\psi, w)$ is the solution of $(4)$. Since $\left(\partial_{n} \psi, \partial_{x_{1}} w(L)\right) \in$ $L^{2}\left(\Sigma_{c}\right) \times L^{2}(0, T)$ and $\left(\psi, \psi^{\prime}, w, w^{\prime}\right) \in \mathcal{C}([0, T] ; Z)$, we can pass to the limit in the above variational formulation. Thus, the pair $(\phi, z)$ is a very weak solution of $(2)$. Moreover, we have

$$
\begin{aligned}
& \int_{0}^{t} \int_{\Omega} f \phi_{n}+\alpha \int_{0}^{t} \int_{\Gamma_{s}} g z_{n} \\
& =\left\langle\phi_{n}^{1}, \psi(0)\right\rangle_{V^{\prime}, V}-\left\langle\phi_{n}^{\prime}(t), \psi(t)\right\rangle_{V^{\prime}, V}-\left(\phi_{n}^{0}, \psi^{\prime}(0)\right)_{L^{2}(\Omega)}+\left(\phi_{n}(t), \psi^{\prime}(t)\right)_{L^{2}(\Omega)} \\
& +\left\langle\alpha z_{n}^{1}+c_{f}^{2} \phi_{n}^{0}, w(0)\right\rangle_{H^{-1}\left(\Gamma_{s}\right), H_{0}^{1}\left(\Gamma_{s}\right)}-\left\langle\alpha z_{n}^{\prime}(t)+c_{f}^{2} \phi_{n}(t), w(t)\right\rangle_{H^{-1}\left(\Gamma_{s}\right), H_{0}^{1}\left(\Gamma_{s}\right)} \\
& -\left(z_{n}^{0}, \alpha w^{\prime}(0)+c_{f}^{2} \psi(0)\right)_{L^{2}\left(\Gamma_{s}\right)}+\left(z_{n}(t), \alpha w^{\prime}(t)+c_{f}^{2} \psi(t)\right)_{L^{2}\left(\Gamma_{s}\right)}-c_{f}^{2} \int_{0}^{t} \int_{\Gamma_{c}} u_{1}^{n} \partial_{n} \psi-\alpha c_{s}^{2} \int_{0}^{t} u_{2}^{n} \partial_{x_{1}} w(L)
\end{aligned}
$$

for all $t \in[0, T]$ and all $\left(\psi, \psi^{\prime}, w, w^{\prime}, f, g\right) \in \mathcal{C}([0, T] ; Z) \times L^{1}\left(0, T ; L^{2}(\Omega)\right) \times L^{1}\left(0, T ; L^{2}\left(\Gamma_{s}\right)\right)$ satisfying $(8)$. We obtain (9) by passing to the limit in the above identity.

To prove the uniqueness of the function $(\phi, z, \tilde{z})$, we take $\left(\phi^{0}, \phi^{1}, z^{0}, z^{1}\right)=(0,0,0,0)$, and $\left(u_{1}, u_{2}\right)=(0,0)$ in system (2). We first obtain that the corresponding solution $(\phi, z)$ is equal to zero. Next, identity (9) implies that $\tilde{z}=0$.

\section{Proof of Theorem 1.11}

To prove Theorem 1.11, we use the Hilbert Uniqueness Method. If we transpose to system (2) the H. U. Method for the Dirichlet boundary control of the wave equation, we have to consider the solution $(\theta, d)$ of the homogeneous problem (1) corresponding to initial conditions $\left(\theta^{0}, \theta^{1}, d^{0}, d^{1}\right)$ in $Z$. 
Next, we have to use $\left.\partial_{n} \theta\right|_{\Sigma_{c}}$ and $\partial_{x_{1}} d(L)$ as controls in the backward problem:

$$
\begin{cases}\psi^{\prime \prime}-c_{f}^{2} \Delta \psi=0 & \text { in } Q, \\ \psi=\partial_{n} \theta & \text { on } \Sigma_{c}, \\ \psi=0 & \text { on } \Sigma_{0} \backslash \Sigma_{c}, \\ \partial_{n} \psi=w^{\prime} & \text { on } \Sigma_{s}, \\ \psi(T)=0 \quad \text { and } \quad \psi^{\prime}(T)=0 & \text { in } \Omega, \\ w^{\prime \prime}-c_{s}^{2} \Delta_{s} w=-\frac{\rho_{f}}{\rho_{s}} \psi^{\prime} & \text { in } \Sigma_{s}, \\ w(L)=\partial_{x_{1}} d(L) \quad \text { and } \quad w(0)=0 & \text { on }(0, T), \\ w(T)=0 \quad \text { and } \quad w^{\prime}(T)=0 & \text { in } \Gamma_{s} .\end{cases}
$$

In Lemma 4.1 , we are going to see that the pairs $(\theta, d)$ and $(\psi, w)$ satisfy a formula of the type:

$$
\begin{aligned}
& -\left\langle\psi^{\prime}(0), \theta(0)\right\rangle_{V^{\prime}, V}+\left(\psi(0), \theta^{\prime}(0)\right)_{L^{2}(\Omega)}-\langle\tilde{w}(0), d(0)\rangle_{H^{-1}\left(\Gamma_{s}\right), H_{0}^{1}\left(\Gamma_{s}\right)} \\
& +\left(w(0), \alpha d^{1}+c_{f}^{2} \theta^{0}\right)_{L^{2}\left(\Gamma_{s}\right)}=c_{f}^{2} \int_{\Sigma_{c}}\left|\partial_{n} \theta\right|^{2}+\alpha c_{s}^{2} \int_{0}^{T}\left|\partial_{x_{1}} d(L)\right|^{2},
\end{aligned}
$$

where $\tilde{w}$ is the unique function in $\mathcal{C}\left([0, T] ; H^{-1}\left(\Gamma_{s}\right)\right)$ satisfying (23) (the analogue of (9) for the adjoint system (21)). In the H. U. Method, to control an intial condition $\left(\phi^{0}, \phi^{1}, z^{0}, z^{1}\right) \in Y$, we look for initial data $\left(\theta^{0}, \theta^{1}, d^{0}, d^{1}\right) \in Z$ so that the solution $(\psi, w)$ of $(21)$ obeys:

$$
\left(\psi(0), \psi^{\prime}(0)\right)=\left(\phi^{0}, \phi^{1}\right) \quad \text { in } \quad \Omega, \quad \text { and } \quad\left(w(0), w^{\prime}(0)\right)=\left(z^{0}, z^{1}\right) \quad \text { on } \quad \Gamma_{s} .
$$

In the classical H. U. Method the existence of such initial data $\left(\theta^{0}, \theta^{1}, d^{0}, d^{1}\right) \in Z$ is obtained by showing that the mapping

$$
\left(\theta^{0}, \theta^{1}, d^{0}, d^{1}\right) \longmapsto\left(\psi^{\prime}(0),-\psi(0), w^{\prime}(0),-w(0)\right)
$$

is an isomorphism from $Z$ to $Z^{\prime} \equiv V^{\prime} \times L^{2}(\Omega) \times H^{-1}\left(\Gamma_{s}\right) \times L^{2}\left(\Gamma_{s}\right)$. Due to formula (22), in our case we have to consider the mapping

$$
\left(\theta^{0}, \theta^{1}, d^{0}, d^{1}\right) \longmapsto\left(\psi^{\prime}(0),-\psi(0), \tilde{w}(0),-w(0)\right) .
$$

Henceforth following the method introduced in [10] for the Helmholtz system, we define the operator $\Lambda$ from $Z$ into $Z^{\prime}$ by

$$
\Lambda\left(\theta^{0}, \theta^{1}, d^{0}, d^{1}\right)=\left(\psi^{\prime}(0),-\psi(0), \tilde{w}(0),-w(0)\right) .
$$

We are going to show that $\Lambda$ is an isomorphism from $Z$ to $Z^{\prime}$, which allows us to prove our controllability result. For that, in Appendix, we prove a direct and an inverse inequality (see Theorem 5.3 and 5.4), which implies that the mapping

$$
\left(\theta^{0}, \theta^{1}, d^{0}, d^{1}\right) \longmapsto\left(c_{f}^{2} \int_{\Sigma_{c}}\left|\partial_{n} \theta\right|^{2}+\alpha c_{s}^{2} \int_{0}^{T}\left|\partial_{x_{1}} d\right|^{2}(L)\right)^{1 / 2}
$$

is a norm on $Z$ for $T$ chosen large enough.

¿From Theorem 1.2, we know that the system (1) admits a unique weak solution $\left(\theta, \theta^{\prime}, d, d^{\prime}\right)$ in $\mathcal{C}([0, T] ; Z)$.

The definition of weak solution of system (21) may be adapted from the one given in Definition 1.5 or in Definition 1.7 for the system (2). As in Theorem 1.9, we can prove that system (21) admits a unique solution $(\psi, w)$ in $L^{\infty}\left(0, T ; L^{2}(\Omega)\right) \times L^{\infty}\left(0, T ; L^{2}\left(\Gamma_{s}\right)\right)$, and that there exists a unique function $\tilde{w} \in \mathcal{C}\left([0, T] ; H^{-1}\left(\Gamma_{s}\right)\right)$ 
satisfying the following formula:

$$
\begin{aligned}
& \int_{t}^{T} \int_{\Omega} f \psi+\alpha \int_{t}^{T} \int_{\Gamma_{s}} g w \\
& =\left\langle\psi^{\prime}(t), \zeta(t)\right\rangle_{V^{\prime}, V}-\left(\psi(t), \zeta^{\prime}(t)\right)_{L^{2}(\Omega)}+\langle\tilde{w}(t), s(t)\rangle_{H^{-1}\left(\Gamma_{s}\right), H_{0}^{1}\left(\Gamma_{s}\right)} \\
& -\left(w(t), \alpha s^{\prime}(t)+c_{f}^{2} \zeta(t)\right)_{L^{2}\left(\Gamma_{s}\right)}-c_{f}^{2} \int_{t}^{T} \int_{\Gamma_{c}} \partial_{n} \theta \partial_{n} \zeta-\alpha c_{s}^{2} \int_{t}^{T} \partial_{x_{1}} d(L) \partial_{x_{1}} s(L),
\end{aligned}
$$

for all $t \in[0, T]$, and all $\left(\zeta, \zeta^{\prime}, s, s^{\prime}, f, g\right)$ belonging to $\mathcal{C}([0, T] ; Z) \times L^{1}\left(0, T ; L^{2}(\Omega)\right) \times L^{1}\left(0, T ; L^{2}\left(\Gamma_{s}\right)\right)$ and obeying (8).

Therefore, setting $t=0$ in the above identity, we see that $(\psi, w)$ is the unique very weak solution of $(2)$ associated with $u_{1}=\partial_{n} \theta, u_{2}=\partial_{x_{1}} d(L)$ and the initial condition $\left(\psi^{0}, \psi^{1}, w^{0}, w^{1}\right)=(\tilde{J})^{-1}\left(\psi(0), \psi^{\prime}(0), w(0), \tilde{w}(0)\right)$.

Before proving that $\Lambda$ is an isomorphism from $Z$ into $Z^{\prime}$, we establish the following lemma.

Lemma 4.1. For all $\left(\phi^{0}, \phi^{1}, z^{0}, z^{1}\right)$ be in $Z$ and all $\left(\theta^{0}, \theta^{1}, d^{0}, d^{1}\right)$ be in $Z$, we have

$$
\left\langle\Lambda\left(\theta^{0}, \theta^{1}, d^{0}, d^{1}\right), J\left(\phi^{0}, \phi^{1}, z^{0}, z^{1}\right)\right\rangle_{Z^{\prime}, Z}=c_{f}^{2} \int_{\Sigma_{c}} \partial_{n} \phi \partial_{n} \theta+\alpha c_{s}^{2} \int_{0}^{T} \partial_{x_{1}} z(L) \partial_{x_{1}} d(L)
$$

where $(\phi, z)$ and $(\theta, d)$ are the solutions of $(1)$ corresponding respectively to $\left(\phi^{0}, \phi^{1}, z^{0}, z^{1}\right)$ and $\left(\theta^{0}, \theta^{1}, d^{0}, d^{1}\right)$.

Proof. The identity in the Lemma is nothing but the formula $(7)$, in which the roles of $(\phi, z)$ and $(\psi, w)$ are permuted:

$$
\begin{aligned}
& \left\langle\psi^{\prime}(0), \phi^{0}\right\rangle_{V^{\prime}, V}-\left(\psi(0), \phi^{1}\right)_{L^{2}(\Omega)}+\left\langle\tilde{w}(0), z^{0}\right\rangle_{H^{-1}\left(\Gamma_{s}\right), H_{0}^{1}\left(\Gamma_{s}\right)} \\
& -\left(w(0), \alpha z^{1}+c_{f}^{2} \phi^{0}\right)_{L^{2}\left(\Gamma_{s}\right)}-c_{f}^{2} \int_{\Sigma_{c}} \partial_{n} \theta \partial_{n} \phi-\alpha c_{s}^{2} \int_{0}^{T} \partial_{x_{1}} d(L) \partial_{x_{1}} z(L)=0 .
\end{aligned}
$$

Up to the end of this section, we suppose that $T>T_{0}$, where $T_{0}$ is defined in Theorem 5.4. We introduce the seminorm in $Z$ defined by

$$
\left\|\left(\theta^{0}, \theta^{1}, d^{0}, d^{1}\right) \mid\right\|_{Z}:=\left(c_{f}^{2} \int_{\Sigma_{c}}\left|\partial_{n} \theta\right|^{2}+\alpha c_{s}^{2} \int_{0}^{T}\left|\partial_{x_{1}} d(L)\right|^{2}\right)^{1 / 2}
$$

where $(\theta, d)$ is the solution of (1) with the initial condition $\left(\theta^{0}, \theta^{1}, d^{0}, d^{1}\right)$. ¿From the direct and inverse inequalities (see Theorems 5.3 and 5.4 in Appendix), it follows that $\left|\|\cdot \mid\|_{Z}\right.$ is a norm in $Z$, equivalent to $\|\cdot\|_{Z}$. We denote by $((\cdot, \cdot))_{Z}$ the associated inner product:

$$
\left(\left(\left(\theta^{0}, \theta^{1}, d^{0}, d^{1}\right),\left(\phi^{0}, \phi^{1}, z^{0}, z^{1}\right)\right)\right)_{Z}:=c_{f}^{2} \int_{\Sigma_{c}} \partial_{n} \theta \partial_{n} \phi+\alpha c_{s}^{2} \int_{0}^{T} \partial_{x_{1}} d(L) \partial_{x_{1}} z(L)
$$

where $(\phi, z)$ and $(\theta, d)$ are the solutions of $(1)$ respectively associated with the initial conditions $\left(\phi^{0}, \phi^{1}, z^{0}, z^{1}\right)$ and $\left(\theta^{0}, \theta^{1}, d^{0}, d^{1}\right)$.

Lemma 4.2. The operator $\Lambda$ is an isomorphism from $Z$ into $Z^{\prime}$.

Proof. Let us first show that $\Lambda$ is continuous from $Z$ into $Z^{\prime}$. Fix $\left(\theta^{0}, \theta^{1}, d^{0}, d^{1}\right) \in Z$. Let us denote $(\theta, d)$ the associated solution of (1). Due to Lemma 4.1, for all $\left(\phi^{0}, \phi^{1}, z^{0}, z^{1}\right) \in Z$, we have

$$
\left\langle\Lambda\left(\theta^{0}, \theta^{1}, d^{0}, d^{1}\right),\left(\phi^{0}, \phi^{1}, z^{0}, z^{1}\right)\right\rangle_{Z^{\prime}, Z}=\left(\left(\left(\theta^{0}, \theta^{1}, d^{0}, d^{1}\right), J^{-1}\left(\phi^{0}, \phi^{1}, z^{0}, z^{1}\right)\right)\right)_{Z},
$$


where $(\phi, z)$ the solution of (1) associated to $\left(\phi^{0}, \phi^{1}, z^{0}, z^{1}\right)$. Hence, using Lemma 3.2 and the equivalence of the norms $\|\cdot\|_{Z}$ and $\left|\|\cdot \mid\|_{Z}\right.$, it follows that

$$
\begin{aligned}
& \left|\left\langle\Lambda\left(\theta^{0}, \theta^{1}, d^{0}, d^{1}\right),\left(\phi^{0}, \phi^{1}, z^{0}, z^{1}\right)\right\rangle_{Z^{\prime}, Z}\right| \leq\left|\left\|( \theta ^ { 0 } , \theta ^ { 1 } , d ^ { 0 } , d ^ { 1 } ) \left|\left\|_ { Z } \left|\left\|J^{-1}\left(\phi^{0}, \phi^{1}, z^{0}, z^{1}\right) \mid\right\|_{Z}\right.\right.\right.\right.\right. \\
& \leq C\left\|\left(\theta^{0}, \theta^{1}, d^{0}, d^{1}\right)\right\|_{Z}\left\|\left(\phi^{0}, \phi^{1}, z^{0}, z^{1}\right)\right\|_{Z},
\end{aligned}
$$

for all $\left(\phi^{0}, \phi^{1}, z^{0}, z^{1}\right) \in Z$. Therefore, $\Lambda$ is continuous from $Z$ into $Z^{\prime}$.

Let us show that $\Lambda$ is an isomorphism from $Z$ into $Z^{\prime}$. Still with Lemma 4.1, we have:

$$
\left\langle J^{*} \Lambda\left(\theta^{0}, \theta^{1}, d^{0}, d^{1}\right),\left(\phi^{0}, \phi^{1}, z^{0}, z^{1}\right)\right\rangle_{Z^{\prime}, Z}=\left(\left(\left(\theta^{0}, \theta^{1}, d^{0}, d^{1}\right),\left(\phi^{0}, \phi^{1}, z^{0}, z^{1}\right)\right)\right)_{Z},
$$

for all $\left(\theta^{0}, \theta^{1}, d^{0}, d^{1}\right),\left(\phi^{0}, \phi^{1}, z^{0}, z^{1}\right)$ in $Z$. ¿From Riesz's Theorem we deduce that $J^{*} \Lambda$ is an isomorphism from $Z$ into $Z^{\prime}$. Thus, $\Lambda=\left(J^{*}\right)^{-1}\left(J^{*} \Lambda\right)$ is an isomorphism from $Z$ into $Z^{\prime}$.

Proof of Theorem 1.11. Let $\left(\phi^{0}, \phi^{1}, z^{0}, z^{1}\right)$ be in $Y$. Then $\tilde{J}\left(\phi^{0}, \phi^{1}, z^{0}, z^{1}\right)=\left(\phi^{0}, \phi^{1}, z^{0}, \tilde{z}^{1}\right)$ belongs to $L^{2}(\Omega) \times$ $V^{\prime} \times L^{2}(\Omega) \times H^{-1}\left(\Gamma_{s}\right)$, and $\left(\phi^{1},-\phi^{0}, \tilde{z}^{1},-z^{0}\right) \in Z^{\prime}$. Since $\Lambda$ is an isomorphism from $Z$ to $Z^{\prime}$, there exists $\left(\theta^{0}, \theta^{1}, d^{0}, d^{1}\right) \in Z$ such that

$$
\Lambda\left(\theta^{0}, \theta^{1}, d^{0}, d^{1}\right)=\left(\phi^{1},-\phi^{0}, \tilde{z}^{1},-z^{0}\right) .
$$

Let us denote $(\theta, d)$ the weak solution of the homogeneous problem (1) with the initial condition $\left(\theta^{0}, \theta^{1}, d^{0}, d^{1}\right)$. The solution $(\psi, w)$ of the backward problem (21) is the unique very weak solution of (2) associated with $u_{1}=\partial_{n} \theta, u_{2}=\partial_{x_{1}} d(L)$ and the initial condition $\left(\psi^{0}, \psi^{1}, w^{0}, w^{1}\right)=(\tilde{J})^{-1}\left(\psi(0), \psi^{\prime}(0), w(0), \tilde{w}(0)\right)$. By definition of $\Lambda$, we have

$$
\Lambda\left(\theta^{0}, \theta^{1}, d^{0}, d^{1}\right)=\left(\psi^{1},-\psi^{0}, \tilde{w}^{1},-w^{0}\right)
$$

Thus equality (24) implies that

$$
\tilde{J}\left(\psi^{0}, \psi^{1}, w^{0}, w^{1}\right)=\tilde{J}\left(\phi^{0}, \phi^{1}, z^{0}, z^{1}\right) .
$$

Therefore, setting $u_{1}=\partial_{n} \theta$ and $u_{2}=\partial_{x_{1}} d(L),(\psi, w)$ is solution of our controllability problem.

\section{Appendix: technical tools}

The direct and inverse inequalities are not obvious and their proof are technical. It is based on a lemma obtained by the multiplier method.

\subsection{Multiplier Lemma}

Consider the following nonhomogeneous problem:

$$
\begin{cases}\phi^{\prime \prime}-c_{f}^{2} \Delta \phi=f & \text { in } Q, \\ \phi=0 & \text { on } \Sigma_{0}, \\ \partial_{n} \phi=z^{\prime} & \text { on } \Sigma_{s}, \\ \phi(0)=\phi^{0} \quad \text { and } \quad \phi^{\prime}(0)=\phi^{1} & \text { in } \Omega, \\ z^{\prime \prime}-c_{s}^{2} \Delta_{s} z=-\frac{\rho_{f}}{\rho_{s}} \phi^{\prime}+g & \text { in } \Sigma_{s}, \\ z=0 & \text { on } \partial \Gamma_{s} \times(0, T), \\ z(0)=z^{0} \quad \text { and } \quad z^{\prime}(0)=z^{1} & \text { in } \Gamma_{s} .\end{cases}
$$


Lemma 5.1. Let $\left(\phi^{0}, \phi^{1}, z^{0}, z^{1}\right)$ be in $D(A),(f, g) \in W^{1,1}\left(0, T ; L^{2}(\Omega) \times L^{2}\left(\Gamma_{s}\right)\right)$, and let $(\phi, z)$ be the corresponding solution of problem $(25)$. Let $q=\left(q_{1}, q_{2}\right)$ be in $C^{1}(\bar{\Omega})^{2}$ and $p$ be in $C^{1}\left(\bar{\Gamma}_{s}\right)$. Then the following identities hold:

$$
\begin{aligned}
& \frac{c_{f}^{2}}{2} \int_{\Sigma_{0}} q \cdot n\left|\partial_{n} \phi\right|^{2} \\
& =\left[\int_{\Omega} \phi^{\prime} q \cdot \nabla \phi\right]_{0}^{T}+\frac{1}{2} \int_{Q} \operatorname{div}(q)\left(\phi^{\prime 2}-c_{f}^{2}|\nabla \phi|^{2}\right)+c_{f}^{2} \sum_{j=1,2} \int_{Q} \partial_{x_{j}} \phi \partial_{x_{j}} q \cdot \nabla \phi \\
& -\frac{1}{2} \int_{\Sigma_{s}} q \cdot n\left(\phi^{\prime 2}-c_{f}^{2}|\nabla \phi|^{2}\right)-c_{f}^{2} \int_{\Sigma_{s}} z^{\prime} q \cdot \nabla \phi-\int_{Q} f q \cdot \nabla \phi,
\end{aligned}
$$

and

$$
\frac{c_{s}^{2}}{2} \int_{0}^{T}\left[p\left|\partial_{x_{1}} z\right|^{2}\right]_{0}^{L}=\left[\int_{\Gamma_{s}} z^{\prime} p \cdot \nabla_{s} z\right]_{0}^{T}+\frac{1}{2} \int_{\Sigma_{s}} \frac{d p}{d x_{1}}\left(z^{\prime 2}+c_{s}^{2}\left|\nabla_{s} z\right|^{2}\right)+\frac{\rho_{f}}{\rho_{s}} \int_{\Sigma_{s}} \phi^{\prime} p \cdot \nabla_{s} z-\int_{\Sigma_{s}} g p \cdot \nabla_{s} z .
$$

Proof. We first prove (26). Since $\left(\phi^{0}, \phi^{1}, z^{0}, z^{1}\right) \in D(A)$ and $(f, g) \in W^{1,1}\left(0, T ; L^{2}(\Omega) \times L^{2}\left(\Gamma_{s}\right)\right),(\phi, z)$ belongs to $L^{\infty}\left(0, T ; H^{2}(\Omega) \times H^{2}\left(\Gamma_{s}\right)\right)$. This smoothness result allows us to justify all of the computations in the proof below.

Multiplying by $q \cdot \nabla \phi$ the equation satisfied by $\phi$ we get:

$$
\int_{Q}\left(\phi^{\prime \prime}-c_{f}^{2} \Delta \phi\right) q \cdot \nabla \phi=\int_{Q} f q \cdot \nabla \phi
$$

With an integration by parts and a Green formula, we obtain:

$$
\begin{aligned}
& \int_{Q} \phi^{\prime \prime} q \cdot \nabla \phi=\left[\int_{\Omega} \phi^{\prime} q \cdot \nabla \phi\right]_{0}^{T}-\int_{Q} \phi^{\prime} q \cdot \nabla \phi^{\prime}=\left[\int_{\Omega} \phi^{\prime} q \cdot \nabla \phi\right]_{0}^{T}-\frac{1}{2} \int_{Q} q \cdot \nabla\left|\phi^{\prime}\right|^{2} \\
& =\left[\int_{\Omega} \phi^{\prime} q \cdot \nabla \phi\right]_{0}^{T}+\frac{1}{2} \int_{Q}\left|\phi^{\prime}\right|^{2} \operatorname{div}(q)-\frac{1}{2} \int_{\Sigma_{s}} q \cdot n\left|\phi^{\prime}\right|^{2}
\end{aligned}
$$

On the other hand, since $\phi \in H^{2}(\Omega)$, with a Green formula, we can write:

$$
\begin{aligned}
& \int_{Q} \Delta \phi q \cdot \nabla \phi=\int_{\Sigma} \partial_{n} \phi q \cdot \nabla \phi-\int_{Q} \nabla \phi \cdot \nabla(q \cdot \nabla \phi) \\
& =\int_{\Sigma} \partial_{n} \phi q \cdot \nabla \phi-\sum_{j=1,2} \int_{Q} \partial_{x_{j}} \phi\left(q \cdot \partial_{x_{j}} \nabla \phi+\partial_{x_{j}} q \cdot \nabla \phi\right) \\
& =\int_{\Sigma} \partial_{n} \phi q \cdot \nabla \phi-\frac{1}{2} \sum_{j=1,2} \int_{Q} q \cdot \nabla\left|\partial_{x_{j}} \phi\right|^{2}-\sum_{j=1,2} \int_{Q} \partial_{x_{j}} \phi \partial_{x_{j}} q \cdot \nabla \phi \\
& =\int_{\Sigma} \partial_{n} \phi q \cdot \nabla \phi+\frac{1}{2} \int_{Q} \operatorname{div}(q)|\nabla \phi|^{2}-\frac{1}{2} \int_{\Sigma} q \cdot n|\nabla \phi|^{2}-\sum_{j=1,2} \int_{Q} \partial_{x_{j}} \phi \partial_{x_{j}} q \cdot \nabla \phi
\end{aligned}
$$


Thus, from (28), (29) and (30), we deduce:

$$
\begin{aligned}
& {\left[\int_{\Omega} \phi^{\prime} q \cdot \nabla \phi\right]_{0}^{T}+\frac{1}{2} \int_{Q} \operatorname{div}(q)\left|\phi^{\prime}\right|^{2}-\frac{1}{2} \int_{\Sigma_{s}} q \cdot n\left|\phi^{\prime}\right|^{2}-c_{f}^{2} \int_{\Sigma} \partial_{n} \phi q \cdot \nabla \phi} \\
& -\frac{c_{f}^{2}}{2} \int_{Q} \operatorname{div}(q)|\nabla \phi|^{2}+\frac{c_{f}^{2}}{2} \int_{\Sigma} q \cdot n|\nabla \phi|^{2}+c_{f}^{2} \sum_{j=1,2} \int_{Q} \partial_{x_{j}} \phi \partial_{x_{j}} q \cdot \nabla \phi=\int_{Q} f q \cdot \nabla \phi .
\end{aligned}
$$

Moreover, since $\phi=0$ on $\Gamma_{0}$, we have:

$$
\nabla \phi=\partial_{n} \phi n \text { on } \Gamma_{0}
$$

and therefore

$$
\begin{aligned}
& \frac{c_{f}^{2}}{2} \int_{\Sigma} q \cdot n|\nabla \phi|^{2}-c_{f}^{2} \int_{\Sigma} \partial_{n} \phi q \cdot \nabla \phi \\
& =\frac{c_{f}^{2}}{2} \int_{\Sigma_{0}} q \cdot n\left|\partial_{n} \phi\right|^{2}+\frac{c_{f}^{2}}{2} \int_{\Sigma_{s}} q \cdot n|\nabla \phi|^{2}-c_{f}^{2} \int_{\Sigma_{0}} q \cdot n\left|\partial_{n} \phi\right|^{2}-c_{f}^{2} \int_{\Sigma_{s}} z^{\prime} q \cdot \nabla \phi \\
& =-\frac{c_{f}^{2}}{2} \int_{\Sigma_{0}} q \cdot n\left|\partial_{n} \phi\right|^{2}+c_{f}^{2} \int_{\Sigma_{s}}\left(\frac{1}{2} q \cdot n|\nabla \phi|^{2}-z^{\prime} q \cdot \nabla \phi\right) .
\end{aligned}
$$

Finally, (26) may be deduced from (31) and (32).

Notice that identity (27) may be obtained in a similar way. The proof of Lemma 5.1 is complete.

In the following we denote by $C$ various positive constants independent of $T$, and by $C_{T}$ constants depending on $T$.

\subsection{Generalized Direct inequality}

In the theorem below we extend to system (25), the hidden regularity result well known for the wave equation.

Theorem 5.2. There exists $C_{T}>0$, only depending of $T$, such that, for all $\left(\phi^{0}, \phi^{1}, z^{0}, z^{1}\right) \in Z$ and all $(f, g) \in L^{1}\left(0, T ; L^{2}(\Omega) \times L^{2}\left(\Gamma_{s}\right)\right)$, the weak solution $(\phi, z)$ of $(25)$ satisfies:

$$
c_{f}^{2} \int_{\Sigma_{c}}\left|\partial_{n} \phi\right|^{2}+\alpha c_{s}^{2} \int_{0}^{T}\left|\partial_{x_{1}} z(L)\right|^{2} \leq C_{T}\left\{\left\|\left(\phi, \phi^{\prime}, z, z^{\prime}\right)\right\|_{L^{\infty}(0, T ; Z)}^{2}+\|f\|_{L^{1}\left(0, T ; L^{2}(\Omega)\right)}^{2}+\alpha\|g\|_{L^{1}\left(0, T ; L^{2}\left(\Gamma_{s}\right)\right)}^{2}\right\} .
$$

Besides, the function $T \mapsto C_{T}$ is nondecreasing.

Proof. Observe that, for weak solutions $\left(\phi, \phi^{\prime}, z, z^{\prime}\right) \in \mathcal{C}([0, T] ; Z)$ of $(25)$, the terms $\left.\partial_{n} \phi\right|_{\Gamma_{c}}$ and $\partial_{x_{1}} z(L)$ are a priori not defined. Therefore, as for the wave equation, we first prove the estimate (33) for classical solutions of (25), that is in the case when $\left(\phi^{0}, \phi^{1}, z^{0}, z^{1}\right) \in D(A)$ and $(0, f, 0, g) \in W^{1,1}(0, T ; Z)$. Next $\left(\left.\partial_{n} \phi\right|_{\Gamma_{c}}, \partial_{x_{1}} z(L)\right)$ is defined as the limit in $L^{2}\left(\Sigma_{c}\right) \times L^{2}(0, T)$ of a sequence $\left(\left(\left.\partial_{n} \phi_{k}\right|_{\Gamma_{c}}, \partial_{x_{1}} z_{k}(L)\right)\right)_{k}$, where $\left(\phi_{k}, z_{k}\right)$ is the classical solution of $(25)$ associated with $\left(\phi_{k}^{0}, \phi_{k}^{1}, z_{k}^{0}, z_{k}^{1}\right) \in D(A)$ and $\left(0, f_{k}, 0, g_{k}\right) \in W^{1,1}(0, T ; Z)$, and where $\left(\left(\phi_{k}^{0}, \phi_{k}^{1}, z_{k}^{0}, z_{k}^{1}\right)\right)_{k}$ converges to $\left(\phi^{0}, \phi^{1}, z^{0}, z^{1}\right)$ in $Z,\left(f_{k}\right)_{k}$ converges to $f$ in $L^{1}\left(0, T ; L^{2}(\Omega)\right),\left(g_{k}\right)_{k}$ converges to $g$ in $L^{1}\left(0, T ; L^{2}\left(\Gamma_{s}\right)\right)$.

Let $(\phi, z)$ be a classical solution of $(25)$. We set $m(x)=x$ for all $x \in \bar{\Omega}$. Notice that there exists $0<\delta \leq L$ such that

$$
m \cdot n \geq \delta \text { on } \Gamma_{c}, \quad m \cdot n=0 \text { on } \Gamma_{0} \backslash \Gamma_{c}, \quad m \cdot n=0 \text { on } \Gamma_{s} .
$$

Let us apply Lemma 5.1 with

$$
q=m \quad \text { and } \quad p\left(x_{1}\right)=x_{1} \quad \forall x_{1} \in[0, L] .
$$


Let us successively estimate the terms in the identity (26). Using the fact that $m \cdot n=0$ on $\Gamma_{s}$, we obtain:

$$
\begin{aligned}
& {\left[\int_{\Omega} \phi^{\prime} m \cdot \nabla \phi\right]_{0}^{T} \leq 2 C\left\|\left(\phi, \phi^{\prime}, z, z^{\prime}\right)\right\|_{L^{\infty}(0, T ; Z)}^{2},} \\
& \frac{1}{2} \int_{Q} \operatorname{div}(m)\left(\phi^{\prime 2}-c_{f}^{2}|\nabla \phi|^{2}\right) \leq C T\left\|\left(\phi, \phi^{\prime}, z, z^{\prime}\right)\right\|_{L^{\infty}(0, T ; Z)}^{2}, \\
& c_{f}^{2} \sum_{j=1,2} \int_{Q} \partial_{x_{j}} \phi \partial_{x_{j}} m \cdot \nabla \phi \leq C T\left\|\left(\phi, \phi^{\prime}, z, z^{\prime}\right)\right\|_{L^{\infty}(0, T ; Z)}^{2}, \\
& \frac{1}{2} \int_{\Sigma_{s}} m \cdot n\left(\phi^{\prime 2}-c_{f}^{2}|\nabla \phi|^{2}\right)=0,
\end{aligned}
$$

and

$$
\int_{Q} f m \cdot \nabla \phi \leq C\left\{\|f\|_{L^{1}\left(0, T ; L^{2}(\Omega)\right)}^{2}+\left\|\left(\phi, \phi^{\prime}, z, z^{\prime}\right)\right\|_{L^{\infty}(0, T ; Z)}^{2}\right\}
$$

Since $m \cdot n \geq \delta$ on $\Gamma_{c}$ and $m \cdot n=0$ on $\Gamma_{0} \backslash \Gamma_{c}$, from (26) and the above estimates, it follows that:

$$
\delta \frac{c_{f}^{2}}{2} \int_{\Sigma_{c}}\left|\partial_{n} \phi\right|^{2} \leq C_{T}\left\{\left\|\left(\phi, \phi^{\prime}, z, z^{\prime}\right)\right\|_{L^{\infty}(0, T ; Z)}^{2}+\|f\|_{L^{1}\left(0, T ; L^{2}(\Omega)\right)}^{2}\right\}-c_{f}^{2} \int_{\Sigma_{s}} z^{\prime} m \cdot \nabla \phi
$$

Similarly, we estimate the terms in the identity (27), and we obtain:

$$
\begin{aligned}
& \alpha\left[\int_{\Gamma_{s}} z^{\prime} p \cdot \nabla_{s} z\right]_{0}^{T} \leq 2 C\left\|\left(\phi, \phi^{\prime}, z, z^{\prime}\right)\right\|_{L^{\infty}(0, T ; Z)}^{2}, \\
& \frac{\alpha}{2} \int_{\Sigma_{s}} \partial_{x_{1}} p\left(z^{\prime 2}+c_{s}^{2}\left|\nabla_{s} z\right|^{2}\right) \leq C T\left\|\left(\phi, \phi^{\prime}, z, z^{\prime}\right)\right\|_{L^{\infty}(0, T ; Z)}^{2}, \\
& \alpha\left|\int_{\Sigma_{s}} g p \cdot \nabla_{s} z\right| \leq C\left\{\alpha\|g\|_{L^{1}\left(0, T ; L^{2}\left(\Gamma_{s}\right)\right)}^{2}+\left\|\left(\phi, \phi^{\prime}, z, z^{\prime}\right)\right\|_{L^{\infty}(0, T ; Z)}^{2}\right\} .
\end{aligned}
$$

Since $p(L)=L \geq \delta$ and $p(0)=0$, it follows that:

$$
\delta \frac{\alpha c_{s}^{2}}{2} \int_{0}^{T}\left|\partial_{x_{1}} z(L)\right|^{2} \leq C_{T}\left\{\left\|\left(\phi, \phi^{\prime}, z, z^{\prime}\right)\right\|_{L^{\infty}(0, T ; Z)}^{2}+\alpha\|g\|_{L^{1}\left(0, T ; L^{2}\left(\Gamma_{s}\right)\right)}^{2}\right\}+c_{f}^{2} \int_{\Sigma_{s}} \phi^{\prime} p \cdot \nabla_{s} z .
$$

The estimates (36) and (37) imply that:

$$
\begin{aligned}
& \delta\left[\frac{c_{f}^{2}}{2} \int_{\Sigma_{c}}\left|\partial_{n} \phi\right|^{2}+\frac{\alpha c_{s}^{2}}{2} \int_{0}^{T}\left|\partial_{x_{1}} z(L)\right|^{2}\right] \\
& \leq C\left\{\left\|\left(\phi, \phi^{\prime}, z, z^{\prime}\right)\right\|_{L^{\infty}(0, T ; Z)}^{2}+\|f\|_{L^{1}\left(0, T ; L^{2}(\Omega)\right)}^{2}+\alpha\|g\|_{L^{1}\left(0, T ; L^{2}\left(\Gamma_{s}\right)\right)}^{2}\right\}+c_{f}^{2} \int_{\Sigma_{s}}\left(\phi^{\prime} p \cdot \nabla_{s} z-z^{\prime} m \cdot \nabla \phi\right) .
\end{aligned}
$$


Moreover, recalling that $\nabla_{s} z=\partial_{x_{1}} z, z=0$ on $\partial \Gamma_{s}$ and $p\left(x_{1}\right)=x_{1}=m\left(x_{1}, 0\right)$ for all $x_{1} \in[0, L]$, we can write:

$$
\begin{aligned}
& \int_{\Sigma_{s}}\left(\phi^{\prime} p \cdot \nabla_{s} z-z^{\prime} m \cdot \nabla \phi\right) \\
& =-\int_{\Sigma_{s}} \phi p \partial_{x_{1}} z^{\prime}+\left[\int_{\Gamma_{s}} \phi p \partial_{x_{1}} z\right]_{0}^{T}-\int_{\Sigma_{s}} z^{\prime} p \partial_{x_{1}} \phi \\
& =\int_{\Sigma_{s}}\left(\partial_{x_{1}} \phi p+\phi \partial_{x_{1}} p\right) z^{\prime}+\left[\int_{\Gamma_{s}} \phi p \partial_{x_{1}} z\right]_{0}^{T}-\int_{\Sigma_{s}} z^{\prime} p \partial_{x_{1}} \phi \\
& =\int_{\Sigma_{s}} \phi \partial_{x_{1}} p z^{\prime}+\left[\int_{\Gamma_{s}} \phi p \partial_{x_{1}} z\right]_{0}^{T} \leq C(T+1)\left\|\left(\phi, \phi^{\prime}, z, z^{\prime}\right)\right\|_{L^{\infty}(0, T ; Z)}^{2}
\end{aligned}
$$

Therefore, there exists a constant $C_{T}>0$ such that the function $T \mapsto C_{T}$ is nondecreasing, and for which we have:

$$
\frac{c_{f}^{2}}{2} \int_{\Sigma_{c}}\left|\partial_{n} \phi\right|^{2}+\frac{\alpha c_{s}^{2}}{2} \int_{0}^{T}\left|\partial_{x_{1}} z(L)\right|^{2} \leq C_{T}\left\{\left\|\left(\phi, \phi^{\prime}, z, z^{\prime}\right)\right\|_{L^{\infty}(0, T ; Z)}^{2}+\|f\|_{L^{1}\left(0, T ; L^{2}(\Omega)\right)}^{2}+\alpha\|g\|_{L^{1}\left(0, T ; L^{2}\left(\Gamma_{s}\right)\right)}^{2}\right\},
$$

which proves (33) for classical solutions.

\subsection{Direct inequality}

Theorem 5.3. For every $T>0$, there exists $C_{T}>0$ such that, for all $\left(\phi^{0}, \phi^{1}, z^{0}, z^{1}\right) \in Z$, the weak solution $(\phi, z)$ of (1) satisfies:

$$
c_{f}^{2} \int_{\Sigma_{c}}\left|\partial_{n} \phi\right|^{2}+\alpha c_{s}^{2} \int_{0}^{T}\left|\partial_{x_{1}} z(L)\right|^{2} \leq C_{T} E(0) .
$$

Moreover the function $T \mapsto C_{T}$ is nondecreasing.

Proof. If $(f, g)=(0,0)$, the energy of the pair $(\phi, z)$ is constant, that is $E(0)=\frac{1}{2}\left\|\left(\phi, \phi^{\prime}, z, z^{\prime}\right)\right\|_{L^{\infty}(0, T ; Z)}^{2}$, and Theorem 5.3 is a direct consequence of Theorem 5.2.

\subsection{Inverse inequality}

The proof of the inverse inequality is based on the classical multiplier $m(x)=x-x_{0}$ where $x_{0}$ is generally chosen such that $x_{0} \in \Omega$ or $x_{0} \notin \Omega$, according to the area on which the control is applied. In our case, the best choice seems to be $x_{0}=(0,0)$. Moreover, choosing $q$ and $p$ as in (35) simplifies several non classical terms due to the coupling.

Let us remark that $\operatorname{div}(q)=2, \frac{d p}{d x_{1}}=1$, and $\partial_{x_{j}} q_{k}=\delta_{k j}$ for all $j, k=1,2$.

Theorem 5.4. There exists a constant $T_{0}>0$ such that, for all $T>T_{0}$ and all weak solution $(\phi, z)$ of (1), we have the estimate:

$$
\left(T-T_{0}\right) E(0) \leq C\left\{c_{f}^{2} \int_{\Sigma_{c}}\left|\partial_{n} \phi\right|^{2}+\alpha c_{s}^{2} \int_{0}^{T}\left|\partial_{x_{1}} z(L)\right|^{2}\right\}
$$

where $C$ is independent of $T$.

Before proving Theorem 5.4, we first state some technical lemmas. 
Lemma 5.5. Let $\beta>0$ be given. The weak solution $(\phi, z)$ of $(1)$ obeys:

$$
\begin{aligned}
& \frac{1}{2} \int_{Q}\left(\phi^{\prime 2}+\beta c_{f}^{2}|\nabla \phi|^{2}\right)+\frac{\alpha}{2} \int_{\Gamma_{s}}\left(z^{\prime 2}+c_{s}^{2}\left|\nabla_{s} z\right|^{2}\right) \\
& =\frac{c_{f}^{2}}{2} \int_{\Sigma_{0}} q \cdot n\left|\partial_{n} \phi\right|^{2}+\frac{\alpha c_{s}^{2}}{2} \int_{0}^{T} p(L)\left|\partial_{x_{1}} z(L)\right|^{2}-\left[\int_{\Omega} \phi^{\prime} q \cdot \nabla \phi\right]_{0}^{T}-\alpha\left[\int_{\Gamma_{s}} z^{\prime} p \cdot \nabla_{s} z\right]_{0}^{T} \\
& -\frac{1}{2} \int_{Q}\left(\phi^{\prime 2}-\beta c_{f}^{2}|\nabla \phi|^{2}\right)+c_{f}^{2} \int_{\Sigma_{s}} z^{\prime} q \cdot \nabla \phi-c_{f}^{2} \int_{\Sigma_{s}} \phi^{\prime} p \cdot \nabla_{s} z .
\end{aligned}
$$

Proof. Applying Lemma 5.1 with $(f, g)=(0,0)$, and using the fact that $\operatorname{div}(q)=2, \partial_{x_{j}} q_{k}=\delta_{k j}$ and $q \cdot n=$ 0 on $\Gamma_{s}$, we have

$$
\begin{aligned}
& \frac{c_{f}^{2}}{2} \int_{\Sigma_{0}} q \cdot n\left|\partial_{n} \phi\right|^{2}=\left[\int_{\Omega} \phi^{\prime} q \cdot \nabla \phi\right]_{0}^{T}+\int_{Q}\left(\phi^{\prime 2}-c_{f}^{2}|\nabla \phi|^{2}\right)+c_{f}^{2} \int_{Q}|\nabla \phi|^{2} \\
& -\frac{1}{2} \int_{\Sigma_{s}} q \cdot n\left(\phi^{\prime 2}-c_{f}^{2}|\nabla \phi|^{2}\right)-c_{f}^{2} \int_{\Sigma_{s}} z^{\prime} q \cdot \nabla \phi \\
& =\left[\int_{\Omega} \phi^{\prime} q \cdot \nabla \phi\right]_{0}^{T}+\int_{Q} \phi^{\prime 2}-c_{f}^{2} \int_{\Sigma_{s}} z^{\prime} q \cdot \nabla \phi
\end{aligned}
$$

Using $\frac{d p}{d x_{1}}=1$ and $p(0)=0$, we obtain:

$$
\frac{c_{s}^{2}}{2} \int_{0}^{T} p(L)\left|\partial_{x_{1}} z(L)\right|^{2}=\left[\int_{\Gamma_{s}} z^{\prime} p \cdot \nabla_{s} z\right]_{0}^{T}+\frac{1}{2} \int_{\Sigma_{s}}\left(z^{\prime 2}+c_{s}^{2}\left|\nabla_{s} z\right|^{2}\right)+\frac{\rho_{f}}{\rho_{s}} \int_{\Sigma_{s}} \phi^{\prime} p \cdot \nabla_{s} z .
$$

Let $\beta>0$ be given and let us write:

$$
\int_{Q} \phi^{\prime 2}=\frac{1}{2} \int_{Q}\left(\phi^{\prime 2}-\beta c_{f}^{2}|\nabla \phi|^{2}\right)+\frac{1}{2} \int_{Q}\left(\phi^{\prime 2}+\beta c_{f}^{2}|\nabla \phi|^{2}\right)
$$

Then, by adding (39) and (40) multiplied by $\alpha$, and using the above identity in the right hand side of 39, Lemma 5.5 is proved.

Lemma 5.6. Let $\beta>0$ be given. The weak solution $(\phi, z)$ of $(1)$ obeys:

$$
\begin{aligned}
& \frac{1}{2} \int_{Q}\left(\phi^{\prime 2}+\beta c_{f}^{2}|\nabla \phi|^{2}\right)+\frac{\alpha}{2} \int_{\Sigma_{s}}\left(z^{\prime 2}+c_{s}^{2}\left|\nabla_{s} z\right|^{2}\right) \\
& \leq C\left(c_{f}^{2} \int_{\Sigma_{c}}\left|\partial_{n} \phi\right|^{2}+\alpha c_{s}^{2} \int_{0}^{T}\left|\partial_{x_{1}} z(L)\right|^{2}+E(0)\right)+\frac{\beta-1}{2} \int_{Q} \phi^{\prime 2}+c_{f}^{2} \frac{\beta-2}{2} \int_{\Sigma_{s}} z^{\prime} \phi .
\end{aligned}
$$

Proof. Let $\beta>0$ be given. Since $q \cdot n=0$ on $\Gamma_{0} \backslash \Gamma_{c}$, with Lemma 5.5 we deduce:

$$
\begin{aligned}
& \frac{1}{2} \int_{Q}\left(\phi^{\prime 2}+\beta c_{f}^{2}|\nabla \phi|^{2}\right)+\frac{\alpha}{2} \int_{\Gamma_{s}}\left(z^{\prime 2}+c_{s}^{2}\left|\nabla_{s} z\right|^{2}\right) \\
& \leq C\left(c_{f}^{2} \int_{\Sigma_{c}}\left|\partial_{n} \phi\right|^{2}+\alpha c_{s}^{2} \int_{0}^{T}\left|\partial_{x_{1}} z(L)\right|^{2}\right)+\left|\left[\int_{\Omega} \phi^{\prime} q \cdot \nabla \phi\right]_{0}^{T}+\alpha\left[\int_{\Gamma_{s}} z^{\prime} p \cdot \nabla_{s} z\right]_{0}^{T}\right| \\
& -\frac{1}{2} \int_{Q}\left(\phi^{\prime 2}-\beta c_{f}^{2}|\nabla \phi|^{2}\right)+c_{f}^{2} \int_{\Sigma_{s}}\left(z^{\prime} q \cdot \nabla \phi-\phi^{\prime} p \cdot \nabla_{s} z\right) .
\end{aligned}
$$


Let us estimate the different terms appearing in the right hand side of (42). The second term can be estimated as follows:

$$
\left|\left[\int_{\Omega} \phi^{\prime} q \cdot \nabla \phi\right]_{0}^{T}+\alpha\left[\int_{\Gamma_{s}} z^{\prime} p \cdot \nabla_{s} z\right]_{0}^{T}\right| \leq C E(0) .
$$

For the last integral, with an integration by parts in space in the first term and in time in the second term, and with $z=0$ on $\partial \Gamma_{s}$, we obtain:

$$
\begin{aligned}
& \int_{\Sigma_{s}}\left(z^{\prime} q \cdot \nabla \phi-\phi^{\prime} p \cdot \nabla_{s} z\right)=\int_{\Sigma_{s}}\left(z^{\prime} x_{1} \partial_{x_{1}} \phi-\phi^{\prime} x_{1} \partial_{x_{1}} z\right) \\
& =-\int_{\Sigma_{s}}\left(x_{1} \partial_{x_{1}} z^{\prime}+z^{\prime}\right) \phi+\int_{\Sigma_{s}} \phi x_{1} \partial_{x_{1}} z^{\prime}-\left[\int_{\Gamma_{s}} \phi x_{1} \partial_{x_{1}} z\right]_{0}^{T} \\
& =-\int_{\Sigma_{s}} z^{\prime} \phi-\left[\int_{\Gamma_{s}} \phi x_{1} \partial_{x_{1}} z\right]_{0}^{T} \leq-\int_{\Sigma_{s}} z^{\prime} \phi+C E(0) .
\end{aligned}
$$

Therefore, we have

$$
\begin{aligned}
& \frac{1}{2} \int_{Q}\left(\phi^{2}+\beta c_{f}^{2}|\nabla \phi|^{2}\right)+\frac{\alpha}{2} \int_{\Gamma_{s}}\left(z^{\prime 2}+c_{s}^{2}\left|\nabla_{s} z\right|^{2}\right) \\
& \leq C\left(c_{f}^{2} \int_{\Sigma_{c}}\left|\partial_{n} \phi\right|^{2}+\alpha c_{s}^{2} \int_{0}^{T}\left|\partial_{x_{1}} z\right|^{2}(L)+E(0)\right)-\frac{1}{2} \int_{Q} \phi^{\prime 2}+\frac{\beta}{2} c_{f}^{2} \int_{Q}|\nabla \phi|^{2}-c_{f}^{2} \int_{\Sigma_{s}} z^{\prime} \phi .
\end{aligned}
$$

Besides, multiplying by $\phi$ the equation satisfied by $\phi$, we get

$$
0=\int_{Q}\left(\phi^{\prime \prime}-c_{f}^{2} \Delta \phi\right) \phi=\left[\int_{\Omega} \phi^{\prime} \phi\right]_{0}^{T}-\int_{Q} \phi^{\prime 2}-c_{f}^{2} \int_{\Sigma} \partial_{n} \phi \phi+c_{f}^{2} \int_{Q}|\nabla \phi|^{2} .
$$

Thus, with the boundary conditions $\partial_{n} \phi=z^{\prime}$ on $\Gamma_{s}$ and $\phi=0$ on $\Gamma_{0}$, we obtain:

$$
\int_{Q}\left(\phi^{\prime 2}-c_{f}^{2}|\nabla \phi|^{2}\right)=\left[\int_{\Omega} \phi^{\prime} \phi\right]_{0}^{T}-c_{f}^{2} \int_{\Sigma_{s}} \partial_{n} \phi \phi=\left[\int_{\Omega} \phi^{\prime} \phi\right]_{0}^{T}-c_{f}^{2} \int_{\Sigma_{s}} z^{\prime} \phi
$$

Hence

$$
c_{f}^{2} \int_{Q}|\nabla \phi|^{2}=\int_{Q} \phi^{2}+c_{f}^{2} \int_{\Sigma_{s}} z^{\prime} \phi-\left[\int_{\Omega} \phi^{\prime} \phi\right]_{0}^{T} \leq \int_{Q} \phi^{\prime 2}+c_{f}^{2} \int_{\Sigma_{s}} z^{\prime} \phi+C E(0) .
$$

The lemma follows from (44) and (45).

Proof of Theorem 5.4. Taking $\beta=2$ in (41), we first obtain

$$
c_{f}^{2} \int_{Q}|\nabla \phi|^{2}+\frac{\alpha}{2} \int_{\Sigma_{s}}\left(z^{\prime 2}+c_{s}^{2}\left|\nabla_{s} z\right|^{2}\right) \leq C\left(c_{f}^{2} \int_{\Sigma_{c}}\left|\partial_{n} \phi\right|^{2}+\alpha c_{s}^{2} \int_{0}^{T}\left|\partial_{x_{1}} z(L)\right|^{2}+E(0)\right) .
$$

In the same way, taking $\beta=1$ in (41) and next using (46), it follows that

$$
\begin{aligned}
& T E(0) \leq C\left(c_{f}^{2} \int_{\Sigma_{c}}\left|\partial_{n} \phi\right|^{2}+\alpha c_{s}^{2} \int_{0}^{T}\left|\partial_{x_{1}} z(L)\right|^{2}+E(0)\right)-\frac{c_{f}^{2}}{2} \int_{\Sigma_{s}} z^{\prime} \phi \\
& \leq C\left(c_{f}^{2} \int_{\Sigma_{c}}\left|\partial_{n} \phi\right|^{2}+\alpha c_{s}^{2} \int_{0}^{T}\left|\partial_{x_{1}} z(L)\right|^{2}+E(0)+\int_{\Sigma_{s}} z^{\prime 2}+\int_{Q}|\nabla \phi|^{2}\right) \\
& \leq C\left(c_{f}^{2} \int_{\Sigma_{c}}\left|\partial_{n} \phi\right|^{2}+\alpha c_{s}^{2} \int_{0}^{T}\left|\partial_{x_{1}} z(L)\right|^{2}+E(0)\right) .
\end{aligned}
$$


Thus there exists $T_{0}>0$ such that

$$
\left(T-T_{0}\right) E(0) \leq C\left(c_{f}^{2} \int_{\Sigma_{c}}\left|\partial_{n} \phi\right|^{2}+\alpha c_{s}^{2} \int_{0}^{T}\left|\partial_{x_{1}} z(L)\right|^{2}\right) .
$$

Therefore Theorem 5.4 is established.

\section{REFERENCES}

[1] G. Avalos, I. Lasiecka, Exact controllability of structural acoustic interactions, J. Math. Pures Appl., 82 (2003), $1047-1073$.

[2] H. T. Banks, R. C. Smith, Y. Wang, Smart material structures, modeling, estimation and control, Masson, Paris 1996.

[3] H. T. Banks, W. Fang, R. J. Silcox, R. C. Smith, Approximation methods for control of structural acoustics models with piezoceramic actuators, J. Intelligent Material Systems Structures, 4 (1993), pp. 98-116.

[4] C. Bardos, G. Lebeau, J. Rauch, Sharp sufficient for the observation, control and stabilization of waves from boundary, SIAM J. Control Optim. 30 (1992), p. 1024-1065.

[5] L. Cot, J.-P. Raymond, J. Vancostenoble, Exact controllability of an aeroacoustic model with a Neumann and a Dirichlet boundary control, in preparation.

[6] P. Destuynder, E. Gout d'Henin, Existence and uniqueness of a solution to an aeroacoustic model, Chin. Ann. of Math., 23B:1 (2002), 11-24.

[7] E. Gout d'Henin, Ondes de Stonley en interaction fluide-structure, PhD Thesis, Université de Poitiers, 2002.

[8] J.-L. Lions, Contrôlabilité exacte, perturbations et stabilisation de systèmes distribués, Masson, Paris, 1998.

[9] S.-D. Micu, Analisis de un sistema hibrido bidimensional fluido-estructura, Thesis, Universidad de Madrid, 1995.

[10] J.-P. Raymond, M. Vanninathan, Exact controllability in fluid-solid structure: The Helmholtz model, ESAIM: COCV, to appear.

[11] G. Savaré, Regularity and perturbation results for mixed second order elliptic problems, Commun. In Partial Differential Equations, 22, 869-899 (1997).

[12] X. Zhang, E. Zuazua, Stability and control on a model in fluid-structure interaction, Proceedings of the 22th Chinese Control Conference, Yichang, China, Wuhan University of Technology Press, Julio 2003, vol 1, 41-43. 